\title{
An automated design process for short pulse laser driven opacity experiments
}

\author{
M. E. Martin ${ }^{\mathrm{a}, \mathrm{b}, *}$, R. A. London ${ }^{\mathrm{a}}, \mathrm{S} . \mathrm{Goluoglu}^{\mathrm{b}}$, H. D. Whitley ${ }^{\mathrm{a}}$ \\ ${ }^{a}$ Lawrence Livermore National Laboratory \\ P.O. Box 808, Livermore, California, 94550 \\ ${ }^{b}$ Nuclear Engineering Program, Department of Materials Science and Engineering, University of Florida \\ PO Box 116400, Gainesville, Florida, 32611
}

\begin{abstract}
Stellar-relevant conditions can be reached by heating a buried layer target with a short pulse laser. Previous design studies of iron buried layer targets found that plasma conditions are dominantly controlled by the laser energy while the accuracy of the inferred opacity is limited by tamper emission and optical depth effects. We developed a process to simultaneously optimize laser and target parameters to meet a variety of design goals. We explored two sets of design cases: a set focused on conditions relevant to the upper radiative zone of the sun (electron temperatures of 200 to 400 $\mathrm{eV}$ and densities greater than 1/10 of solid density) and a set focused on reaching temperatures consistent with deep within the radiative zone of the sun (500 to $1000 \mathrm{eV}$ ) at a fixed density. We found optimized designs for iron targets and determined that the appropriate dopant, for inferring plasma conditions, depends on the goal temperature: magnesium for up to $300 \mathrm{eV}$, aluminum for 300 to $500 \mathrm{eV}$, and sulfur for 500 to $1000 \mathrm{eV}$. The optimal laser energy and buried layer thickness increase with goal temperature. The accuracy of the inferred opacity is limited to between $11 \%$ and $31 \%$, depending on the design. Overall, short pulse laser heated iron experiments reaching stellar-relevant conditions have been designed with consideration of minimizing tamper emission and optical depth effects while meeting plasma condition and x-ray emission goals.
\end{abstract}

Keywords: opacity, emission, design, optimization, stellar, laser

\section{Introduction}

Short pulse lasers have been used to heat materials to hundreds of eVs at near solid densities [1-12. Xray emission measurements, at such stellar-relevant conditions, may provide insight into existing disagreements between solar models and observations [13 21] by testing the validity of theoretical opacity models. In addition, short pulse heated emission measurements would complement previous absorption measurements that used long pulse lasers [22 28] and pulsed power machines [29 32]. Laser parameters and target dimensions can be optimized to design short pulse experiments that reach a range of stellar-relevant conditions.

We previously examined the effects of short pulse laser irradiance and target dimensions on plasma conditions, $\mathrm{x}-$ ray emission, and the opacity inference for iron buried layers. We found that peak plasma temperature and $\mathrm{x}$ ray emission increase with laser energy and the opacity inference is limited by tamper emission and optical depth effects 33. Our earlier work resulted in an example design that mitigated optical depth and tamper emission effects, but the design was not selected using a formal optimization process. In this paper, we expand upon our work by

\footnotetext{
* Corresponding author

Email address: memartin@llnl.gov (M. E. Martin )
}

implementing an automated process to systemically explore a broad range of laser and target parameters and find optimized designs therein. This allows us to determine globally optimized designs for specific goals, such as plasma temperature and density. We used the process to find optimized designs of buried iron sulfide $\left(\mathrm{FeS}_{2}\right)$ targets. The choice of iron sulfide is motivated by experiments at Atomic Weapons Establishment's Orion Laser Facility, which were originally proposed to use $0.3 \mu \mathrm{m}$ iron sulfide sandwiched between two $3 \mu \mathrm{m}$ layers of parylene$\mathrm{N}{ }^{1}$ The experiments were planned to use a $0.53 \mu \mathrm{m}$ wavelength laser beam with a pulse length of 0.5 ps [34, focused to $20-50 \mu \mathrm{m}$ diameters [8], and laser energies up to 100 $\mathrm{J}$ 34. We explored two sets of design cases using the automated process. The first set focused on the range of plasma conditions relevant to the upper radiative zone of the sun: $T=200 \mathrm{eV}, T=300 \mathrm{eV}$, and $T=400 \mathrm{eV}$, each with $\rho>1 / 10$ of solid density. The second set focused on reaching higher plasma temperatures, relevant to deep within the radiative zone of the sun, at a fixed density: $T=500 \mathrm{eV}, T=750 \mathrm{eV}$, and $T=1000 \mathrm{eV}$, each with $\rho=4.2 \mathrm{~g} / \mathrm{cm}^{3}$. The designs for both sets may be valuable for investigating how iron opacity scales with temperature. In this paper, we discuss the automated design process and its application to these six design cases.

${ }^{1}$ D. Hoarty, personal communication (2017) 
In this paper, we use a simulation methodology (Section 2.3 similar to the methodology in our previous work 33. This methodology assumes 1D geometry, which is most applicable to experiments using a buried microdot target in which the laser focal spot is much larger than the thickness of the buried layer and the microdot diameter. Recent work suggests that for experiments in which the laser focal spot diameter is similar to that of the microdot, the inclusion of radial temperature gradients in analysis is important for fitting measured streaked K-shell spectra 35. Hoarty et al. concluded that radial temperature gradients may be reduced by either using a smaller microdot or increasing the laser focal spot diameter. We recognize that even though our methodology has been shown to predict peak plasma conditions and reasonable x-ray emission durations [33], it does not include 2D effects which may affect the analysis of experimental data. We have included further discussion of simulation assumptions in Section 2.3 and how they may impact the application of this work in Section 4

\section{Automated Design Process}

We developed an automated design process using the LLNL Uncertainty Quantification Pipeline (UQP) 36 38 to manage and analyze ensembles of HYDRA 39] simulations of the target dynamics. Figure 1 shows the process steps. We analyze the ensembles using scripts written in Python [40] and Yorick [41]. In the following sections, we describe the opacity inference equation, the general optimization problem addressed in the design process, the HYDRA simulation methodology, the UQP ensemble management, and the post-ensemble analysis process.

\subsection{Opacity Inference}

In a short pulse experiment, the x-ray emission can be used to infer opacity by inverting the solution to the radiation transfer equation:

$$
\kappa_{\nu}=\frac{-\ln \left(1-\frac{I_{\nu}}{B_{\nu}(T)}\right)}{\rho \Delta l}
$$

where $\kappa_{\nu}$ is the frequency dependent opacity, $I_{\nu}$ is the measured specific intensity, $B_{\nu}(T)$ is the Planck function, $T$ is the inferred electron temperature, and $\rho \Delta l$ is the initial areal density of the target. The opacity inference equation (Eq. (1) assumes constant local thermodynamic equilibrium (LTE) conditions. A dopant material, that will be ionized to its K-shell, is typically included in the buried layer so that the plasma temperature $(T)$ can be inferred from relative line intensities and the the electron density can be inferred from the Stark broadened line widths. The effects of the departure from LTE are typically quantified by comparing LTE and non-LTE models.

\subsection{Optimization Formulation}

We define an ideal design as one in which the simulated inferred opacity $\left(\kappa_{\nu}\right)$ agrees with the opacity supplied to HYDRA $\left(\kappa_{\nu}^{\text {model }}\right)$ and meets design constraints at peak temperature. We have defined a general design optimization procedure that minimizes the average relative difference between inferred and model opacity, while satisfying a number of constraints. The average relative difference is

$$
\sigma_{\kappa_{\nu}}^{\mathrm{rel}}=\frac{a b s\left(\kappa_{\nu}-\kappa_{\nu}^{\text {model }}\right)}{\kappa_{\nu}^{\text {model }}}
$$

and the design constraints are summarized in Table 1 . The design constraint forms are normalized by the relevant design constraint value, indicated with "*", so that the equations are unitless. In each constraint form, the variable indicated as "peak" refers to the appropriate single value metric at peak temperature to compare with the design constraint value. Our constrained optimization problem can be converted to an unconstrained problem by defining a cost function that includes a penalty function [42, 43] for each unsatisfied constraint. We define the cost function as

$$
\beta \sigma_{\kappa_{\nu}}^{\mathrm{rel}}+\Sigma_{i=1}^{m} \phi\left(\alpha_{i}, g_{i}\right)
$$

where $\beta$ is the relative difference coefficient, $\sigma_{\kappa_{\nu}}^{\text {rel }}$ is defined in Eq. (2) and $\phi\left(\alpha_{i}, g_{i}\right)$ are penalty functions with parameters $\left\{\alpha_{i}\right\}$ and constraint functions $\left\{g_{i}\right\}$. We used the following penalty function form in this study:

$$
\phi\left(\alpha_{i}, g_{i}\right)= \begin{cases}0, & g_{i}<0 \\ \alpha_{i} g_{i}^{2}, & g_{i} \geq 0\end{cases}
$$

where the constraint functions $\left\{g_{i}\right\}$ are the left hand side of the constraint forms in Table 1. We set the constraint functions, penalty parameters, and relative difference coefficient for each design case. We calculate the cost function (Eq. (3) ) for each ensemble simulation then use quantitative optimization techniques (Section 2.4.2 to find the design parameters that yield the minimum cost function.

\subsection{HYDRA Simulations}

We used the HYDRA radiation hydrodynamics code for the target simulations included in each ensemble. Our simulation methodology is described in detail in Martin et al. (Ref. 33]). Validation 33] and verification [44] of this methodology were addressed previously. We previously examined the temperature dependence of non-LTE effects on plasma conditions, emissivity, opacity, and x-ray emission 33 . We concluded that for temperatures less than $300 \mathrm{eV}$, non-LTE effects were small but for temperatures larger than $400 \mathrm{eV}$, non-LTE effects were significant and should be included in analysis and design. At intermediate temperatures of 300 to $400 \mathrm{eV}$, non-LTE effects on plasma conditions are small but effects on emissivity, opacity, and time resolved x-ray emission are moderate. Depending on uncertainties, these non-LTE effects may be distinguishable in an experiment. Therefore, all simulations discussed 


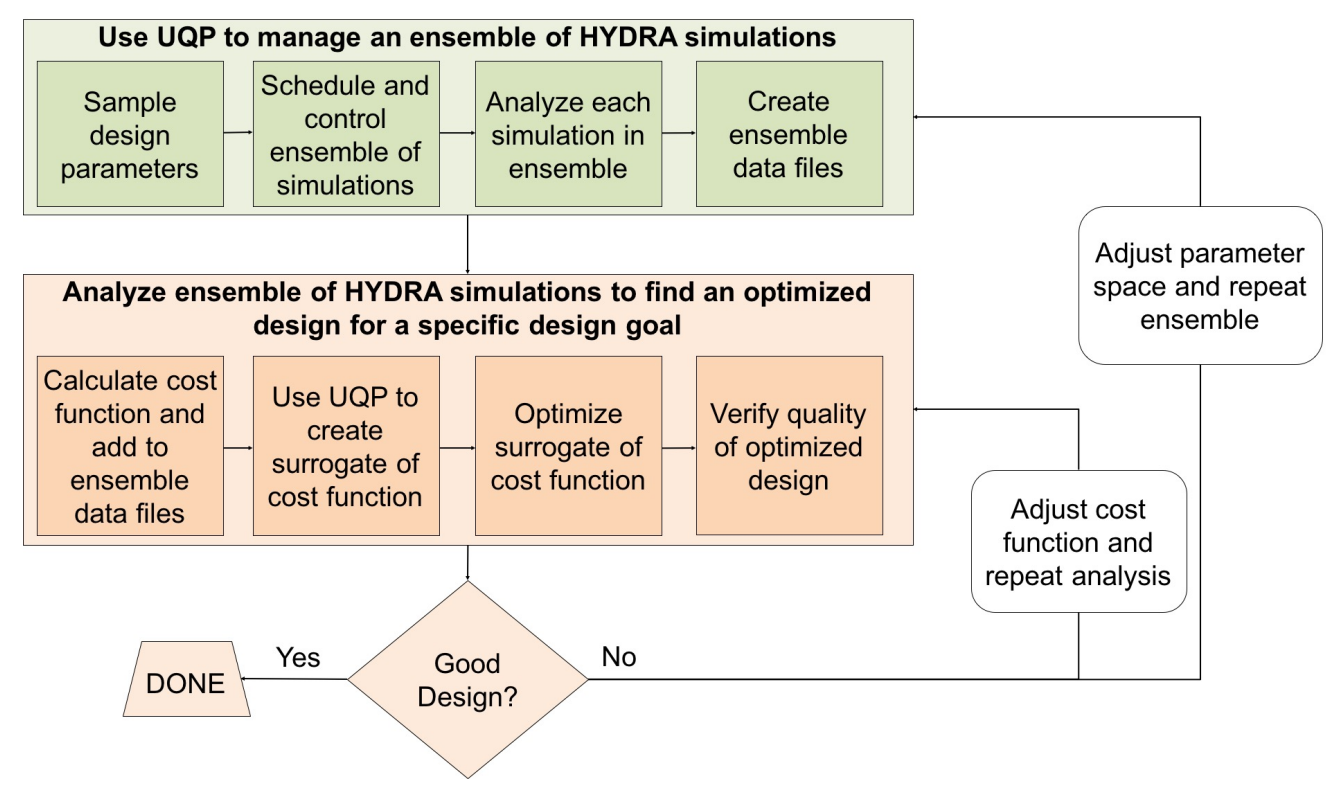

Figure 1: Flow chart of the automated design process we developed.

in this paper used DCA 45, 46] non-LTE atomic models for the opacities and equations of state for electron temperatures larger than $300 \mathrm{eV}$. At temperatures less than $300 \mathrm{eV}$, we assumed DCA LTE opacities, a Thomas-Fermi based [47, 48 equation of state (EOS) for tamper materials and an in-line quotidian equation of state (QEOS) 48 for all buried layer materials. For the $\mathrm{FeS}_{2}$ mixed buried layer, we used an initial density of $4.8 \mathrm{~g} / \mathrm{cm}^{3}$. We assumed a conversion efficiency $(\epsilon)$ of $3 \%$ in our energy source. We simulated a previous Orion Laser Facility short pulse heated buried layer experiment 8 using our energy source with a $3 \%$ conversion efficiency and the results showed good agreement with peak plasma conditions and x-ray pulse durations [33. All details not specifically addressed in this section are the same as in our previous work 33 .

\subsection{Process Flow and Integration with $U Q P$ \\ 2.4.1. UQP ensemble management}

As outlined in Figure 1, we used the UQP to manage ensembles of HYDRA simulations. We first create a general HYDRA input file. We then use the UQP to sample a set of parameters that span our desired space. The UQP manipulates the general HYDRA input file to create an ensemble of specific input files with the selected parameters. The UQP then schedules, launches, and monitors the simulations, including restarting the simulations that failed to finish within the allocated time. Once a simulation is completed, the UQP executes a post-simulation analysis process. In this process, we included a Yorick analysis script that saves HYDRA data to binary files and saves single value metrics, which will later be used to create a cost function for optimization, to text files. Once all of the simulations in the ensemble are completed, the UQP compiles the ensemble results into a set of text files.

\subsubsection{User-controlled ensemble analysis}

As indicated in Figure 1, we separately analyzed the ensemble from the UQP ensemble management. We created a Python script to read the ensemble data file(s) and calculate the cost function given a set of design constraints and the ensemble results. Our Python script adds the design constraints, cost function coefficients, and calculated cost function to the ensemble data files. We used the UQP to create a surrogate of the cost function using Gaussian process regression (GPR) [49]. The purpose of the surrogate is to interpolate the cost function on areas of the parameter space in between ensemble simulations, which allows us optimize across the entire parameter space. In GPR the prediction (estimated cost function values) interpolates the ensemble observations (true cost function values) using a regression function and a Gaussian correlation model. By using GPR, we assume that the correlation is strong between nearby observations and weak between distant observations. We tested the surrogate by comparing the predicted to the true cost function values for a subset of ensemble observations that were not used to train the surrogate. The UQP saves the resulting surrogate model as a Python shelve file [50].

We created another Python script to find the optimal design parameters that minimize the surrogate cost function. We used two existing Python functions to find local minima: basin hopping [51] and brute force [52. Basin hopping evaluates the cost function surrogate for a subset of the parameter space around an initial guess. The smallest surrogate value becomes the new guess. The algorithm evaluates the surrogate for a randomly determined subset of the parameter space around the new guess. If there is another guess with a smaller surrogate value, it is accepted as the new guess and the process is repeated. The process ends when the same guess is found for a number 
of iterations. We repeated basing hopping for at least 100 random initial guesses to find local minima. The local minima were then compared to find the global minimum. As a secondary approach, we used the brute force algorithm. Brute force evaluates the cost function surrogate on a grid within the parameter space that has more points than the number of simulations in the ensemble. The point in the grid with the minimum value is identified as the global minimum. The brute force method is tractable for the small parameter spaces explored in this study. However, as the number of parameters increases, the brute force method will become computationally expensive and may not be tractable.

Once an optimized design is found, we evaluate the quality of that design by executing a HYDRA simulation with the optimized design parameters. We calculate the cost function from the simulation results, then examine the cost function and the constraints. We accept the design if the it meets the constraints sufficiently well. If the design does not meet constraints well, we evaluate why it failed. If the cost function was too sensitive, or not sensitive enough, to a specific design constraint, we adjust the cost function coefficients and repeat the ensemble analysis. For example, if the optimized design reaches a temperature $50 \%$ larger than the goal temperature, the coefficient for the temperature constraint is increased to increase the sensitivity of the cost function to that constraint. If the cost function is adequate, but consideration of the entire ensemble parameter space indicates that the global minimum was not reached, we adjust the parameter space, perform more sampling, and repeat the entire design process.

\section{Results and Analysis}

We used the automated design process to conduct single and multiple design parameter studies of $\mathrm{FeS}_{2}$ buried layer targets. Using our simulation methodology, for a fixed laser pulse length, a given internal energy source can be consistent with multiple combinations of conversion efficiency, laser energy, and laser focal spot size. Therefore, for the parameter studies in this paper, only laser energy and pulse length are considered variable laser parameters. We fixed the conversion efficiency to be $3 \%$, as discussed in Section 2.3, and the laser focal spot size to be $50 \mu \mathrm{m}$ in diameter. The variable target parameters are buried layer and tamper thicknesses. For both single and multiple parameter studies, we considered two sets of design cases: one focused on conditions relevant to the upper radiative zone of the sun and another focused on achieving to higher temperatures. For all design cases, we fixed a subset of the constraint values, which are summarized along with their motivation, in Table 2 .

\subsection{Single Parameter Ensembles}

We generated ensembles varying laser energy $(E=0.1$ - $100 \mathrm{~J})$, laser pulse length $\left(t_{\mathrm{FWHM}}=10 \mathrm{fs}-15 \mathrm{ps}\right)$, buried layer thickness $\left(\Delta l_{\mathrm{BL}}=10 \mathrm{~nm}-1 \mu \mathrm{m}\right)$, and tamper thicknesses $\left(\Delta l_{\mathrm{CH}}=10 \mathrm{~nm}-10 \mu \mathrm{m}\right)$ individually. The laser energy range was selected to match the laser energy range for potential experiments on Orion Laser Facility (Section 1). The laser pulse length, buried layer thickness, and tamper thickness ranges were selected to explore a broader range than previous short pulse laser heated buried layer experiments. We recognize that some regions of this parameter space, such as the combination of high energy and very short pulse length, are unrealistic, being beyond the capability of the current lasers and/or involving very different regimes of laser-plasma interaction. Furthermore, since our methodology has been validated for a narrower subset of the parameter values, any designs outside that subset would need to be validated by comparison to future experiments. However, the optimized designs found in Section 3 are within or near the validated regions of parameter space. For each ensemble, the UQP sampled 20 points uniformly between the upper and lower bounds of the variable parameter. When not being varied, the fixed values of laser energy, laser pulse length, buried layer thickness, and tamper thicknesses were $14 \mathrm{~J}, 0.5 \mathrm{ps}, 0.3 \mu \mathrm{m}$ of $\mathrm{FeS}_{2}$, and $3 \mu \mathrm{m}$ of parylene-N, respectively. We analyzed the ensemble for two sets of design cases with constraint values described in Table 3 . For the design set aimed at reaching higher temperatures at a fixed design density, we changed the minimum density constraint (no. 3) to two inequalities in order to constrain the density within \pm 10 $\%$ of the design density goal:

Constraint no. 3:

$$
\begin{aligned}
& 0.9-\frac{\rho^{\text {peak }}}{\rho^{*}} \leq 0 \\
& \frac{\rho^{\text {peak }}}{\rho^{*}}-1.1 \leq 0
\end{aligned}
$$

For each design case, we determined the cost function minimum by plotting the cost function value versus variable parameter. As an example, Figure 2 shows how the cost function varies with each parameter for the $300 \mathrm{eV}$ design case. For all single parameter ensembles, the 300 $\mathrm{eV}$ design case cost function initially decreases, but then increases with increasing design parameter. Therefore, the optimal design parameter can be visually estimated from the cost function plots. From the scales in Figure 2, we conclude that the cost function is most sensitive to laser energy and target dimensions (buried layer and tamper thickness) and is least sensitive to laser pulse length. The sensitivities of the cost function to laser energy and target dimensions are related to the constraints on the goal temperature and optical depth, respectively. The temperatures reached are dominantly controlled by laser energy while the optical depth is controlled by the buried layer thickness. The tamper thickness also affects the temperatures because, at fixed laser energy, the energy per unit mass decreases as the target mass increases. The general 
behavior described for the $300 \mathrm{eV}$ design case was also seen in the visual analysis of the other design cases listed in Table 3 .

The optimized parameters for each design case are listed in Table 4. For the variable laser energy ensemble, the temperature increases ( $80 \mathrm{eV}$ to $2.1 \mathrm{keV}$ ) with increasing laser energy $(0.1$ to $100 \mathrm{~J})$. The optimal laser energy increased with increasing goal temperature. The variable laser pulse length ensemble reached temperatures ranging from $360 \mathrm{eV}$ to $200 \mathrm{eV}$, for pulse lengths ranging from 10 fs to $15 \mathrm{ps}$. The variable buried layer thickness ensemble reached temperatures ranging from $300 \mathrm{eV}$ to 250 $\mathrm{eV}$ for thicknesses between $10 \mathrm{~nm}$ and $1 \mu \mathrm{m}$. The laser pulse length and buried layer thickness ensembles are not well suited for finding optimized designs for the 400 - 1000 $\mathrm{eV}$ design cases. The variable tamper thickness ensemble reached temperatures between $1.4 \mathrm{keV}$ and $180 \mathrm{eV}$, for tamper thicknesses of $10 \mathrm{~nm}$ to $10 \mu \mathrm{m}$. The decrease in temperature with increasing tamper thickness is because with our energy source, at a fixed laser energy, the deposited energy decreases with increasing tamper thickness. The laser energy ensemble is most applicable to targets proposed for Orion experiments. The optimized design for the laser energy ensemble gives an indication of what laser energies are needed to reach different electron temperatures (assuming $3 \%$ conversion efficiency and $50 \mu \mathrm{m}$ diameter focal spot size). The application of the other single parameter ensembles to Orion experiments is limited. Both the laser pulse length and buried layer thickness ensembles are only applicable to reaching temperatures under $400 \mathrm{eV}$.

\subsection{Two Parameter Ensemble}

We now consider multiple design parameter ensembles in order to further examine the effect of the interplay between parameters on the cost function and optimal designs. We created an ensemble varying laser energy from 0.1 to $100 \mathrm{~J}$ and $\mathrm{FeS}_{2}$ buried layer thickness from $10 \mathrm{~nm}$ to $1 \mu \mathrm{m}$ using the automated design process. We fixed the laser pulse length (0.5 ps) and the tamper thicknesses (3 $\mu \mathrm{m}$ of parylene-N). The UQP managed an ensemble of 768 simulations for parameters selected by Latin Hypercube sampling [53], which provides a pseudo-random sampling of multidimensional spaces. We analyzed the ensemble for the two sets of design cases described in Table 3.

We increased the relative difference coefficient $(\beta)$ and the penalty parameters $\left(\left\{\alpha_{i}\right\}\right)$ until the temperatures of optimized designs where close to the goal temperature. We selected a standard set of coefficients and penalty parameters because the optimized designs became less sensitive to the changes and met most design constraints. We selected the following penalty parameters for the upper radiative zone design set: 100 for the temperature constraints (no. $1 \& 2$ ) and 2 for all other constraints (no. 3 - 17). We selected the same penalty parameters for the higher temperature design set, except we selected 100 for the density constraint (no. 3). For both design sets, we selected 25 for the relative difference coefficient $(\beta)$.

\subsubsection{Surrogate quality}

We created surrogates for each cost function in the upper radiative zone design set $(T=200-400 \mathrm{eV})$, by training the surrogate using 691 of the simulations and testing it using 77 simulations. Training the cost function surrogates, for the higher temperature design set, using the same 691 simulations created poor surrogates because the cost function becomes more sensitive to large optical depths as the goal temperature increases. We removed simulations with large optical depths $(\tau>5)$ in order to create better performing surrogates. This trimmed the edges of the parameter space with high cost function values. The trimmed edges are areas where optimized designs would not be found so removing the edges should still allow local minima to be identified. For the $1000 \mathrm{eV}$ design case, removing the simulations with large optical depths did not yield a high quality surrogate. In addition, we removed simulations with an average relative difference between the inferred and model opacity larger than $100 \%$, which further trimmed the edges of the parameter space with high cost function values. Again, the trimmed edges are areas where an optimized design would not be found.

A summary of the number of simulations used to train and test the surrogate as well as the surrogate quality metrics for each design case are included in Table 5. These quality metrics were calculated for a comparison between the test simulations' true cost function values and the cost function values predicted by the surrogate. The $\mathrm{R}^{2}$ metric is the coefficient of determination 54 and provides the percentage of the variation in the surrogate predictions that can be explained by the relationship between the surrogate and the true cost function. The correlation score [55] gives the percentage of the surrogate predictions that fit a linear response between the surrogate predictions and true cost function values. We defined high quality surrogates as those having a $\mathrm{R}^{2}$ and correlation score larger than $90 \%$. The L-1 [56] relative error is the average absolute relative difference between the surrogate predictions and the true cost function values. For the 200, 300, and $400 \mathrm{eV}$ design cases, the quality of the surrogates decrease as indicated by the decreasing $\mathrm{R}^{2}$ and correlation scores as well as the increasing $\mathrm{L}-1$ relative error. The increasing L-1 relative error suggests that some area of the parameter space is not fit well by the surrogate. Those areas are the edges of the parameter space with large optical depths and thus high cost function values. We could trim those areas to improve the L-1 relative error, but this was not necessary since the surrogate predicts the areas of low cost function values well in spite of these high cost regions.

\subsubsection{Optimized designs}

The basin hopping method found two local minima for each design case surrogate. The brute force method found one global minimum that agreed with one of these local 

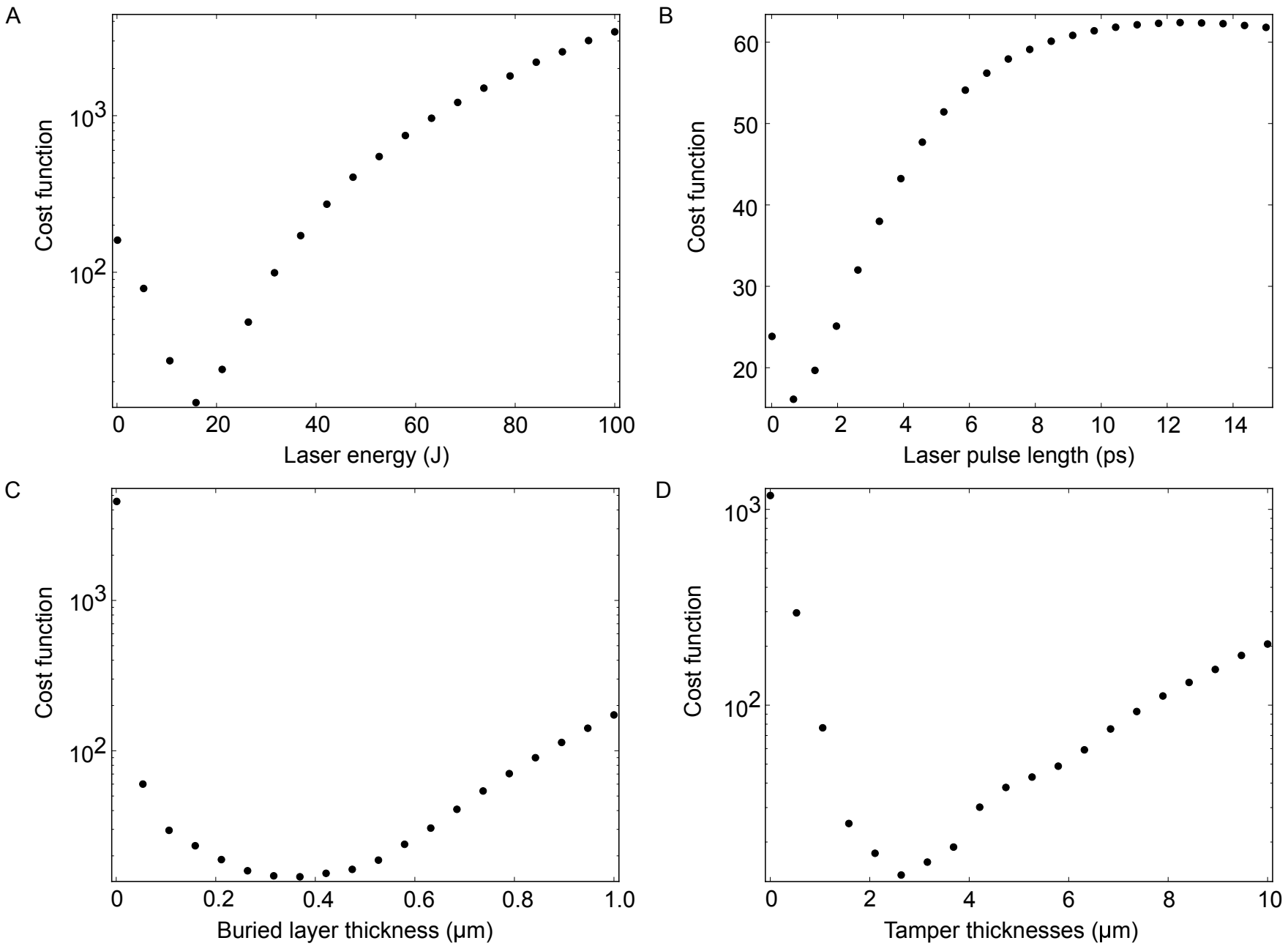

Figure 2: The $300 \mathrm{eV}$ design cost function versus design parameter for four single parameter ensembles. A) Laser energy ensemble, on a log-linear scale. B) Laser pulse length ensemble. C) Buried layer ensemble, on a log-linear scale. D) Tamper thickness ensemble, on a log-linear scale.

minima. Figure 3 shows the cost function surrogate for the $300 \mathrm{eV}$ and $750 \mathrm{eV}$ design cases. The circles indicate the parameter values of the ensemble simulations. The white squares mark the local minima. For all design cases, the quantitative optimization approaches yielded designs that were in the areas where the surrogate values are small, indicating the quantitative approaches successfully identified the areas where local minima occur.

In general, the areas of high cost function surrogate values are dominated by the failure to meet temperature constraints (no. $1 \& 2$ ) and optical depth constraints (no. $7 \& 8$ ). Figure 4 shows the surrogates for peak electron temperature and maximum optical depth over the Fe emission range for the $300 \mathrm{eV}$ design case. The thin white region in Figure $4 \mathrm{~A}$ indicates the area of the parameter space where the temperature constraints are met $(285-315 \mathrm{eV})$. The areas of Figure $4 \mathrm{~B}$ with values between 0.03 and 0.64 meet the optical depth constraints. The white region in Figure $4 \mathrm{~B}$ indicates values between 0.48 - 0.52. Based on the surrogates in Figure 4, we find that neither of the local minima found from the optimization process strictly met the temperature and optical depth constraints. However, the local minimum with a thinner buried layer more closely meets the constraints. The thicker local minimum has a temperature approximately a third higher than the goal $(300 \mathrm{eV})$ and an optical depth larger than 1 . Similar results are found for the other design cases, but the region of the parameter space where the temperature constraints are met shifts with goal temperature, spanning a lower laser energy range for the $200 \mathrm{eV}$ design case and increasingly higher laser energy ranges for the 400 to 1000 $\mathrm{eV}$ design cases. The maximum optical depth surrogate remains similar for the other design cases.

For each local minimum, we executed a HYDRA simulation with the design parameters found by the quantitative optimization. We calculated the single value metrics, constraints, and cost function using these simulation results. We examined the cost function and constraints to determine which of the minima best satisfies the design constraints. For all design cases, the optimization yields one minimum with a lower laser energy and thinner buried layer and one with a higher laser energy and thicker 
A

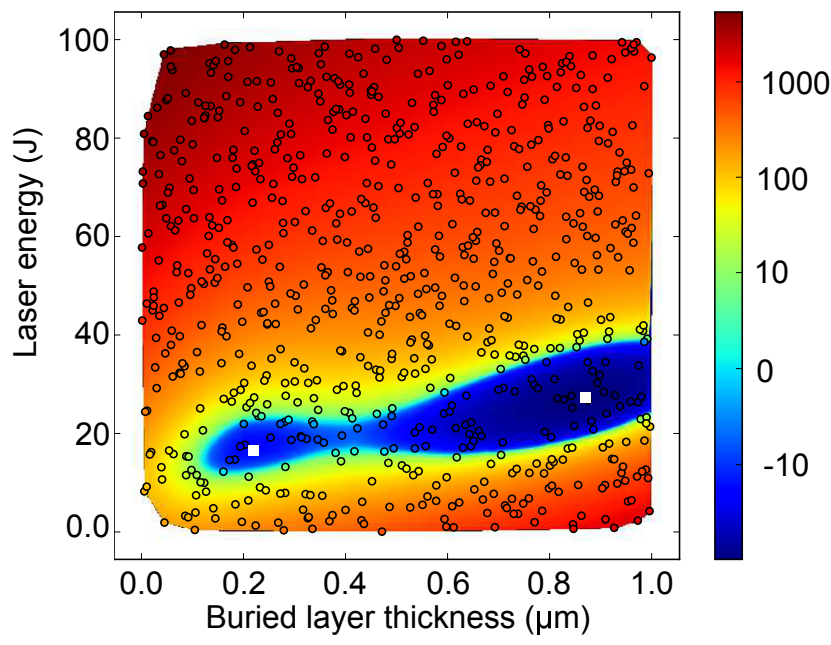

B

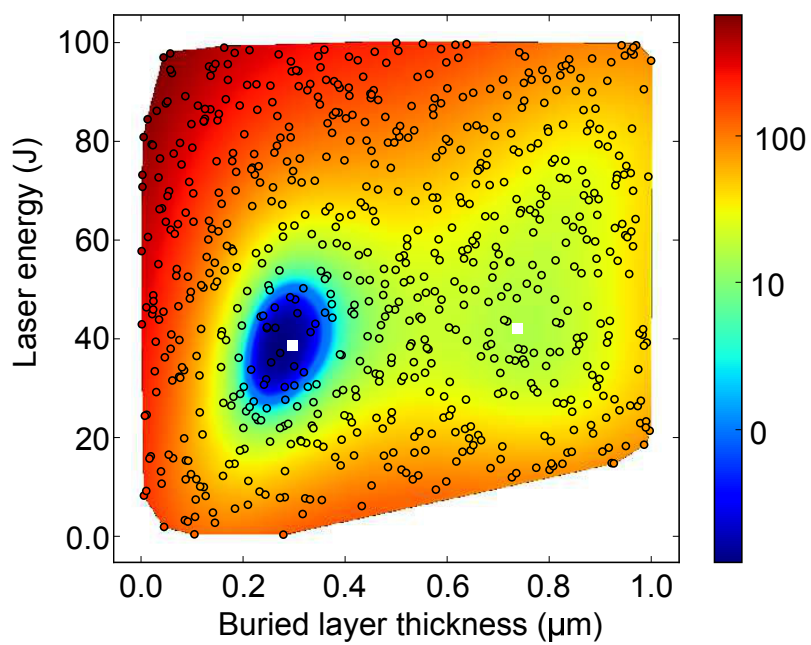

Figure 3: Cost function surrogates for two design cases. The circles mark the design parameter values of the ensemble simulations. The white squares mark the design parameter values of the local minima. A) $300 \mathrm{eV}$ design case B) $750 \mathrm{eV}$ design case.

A

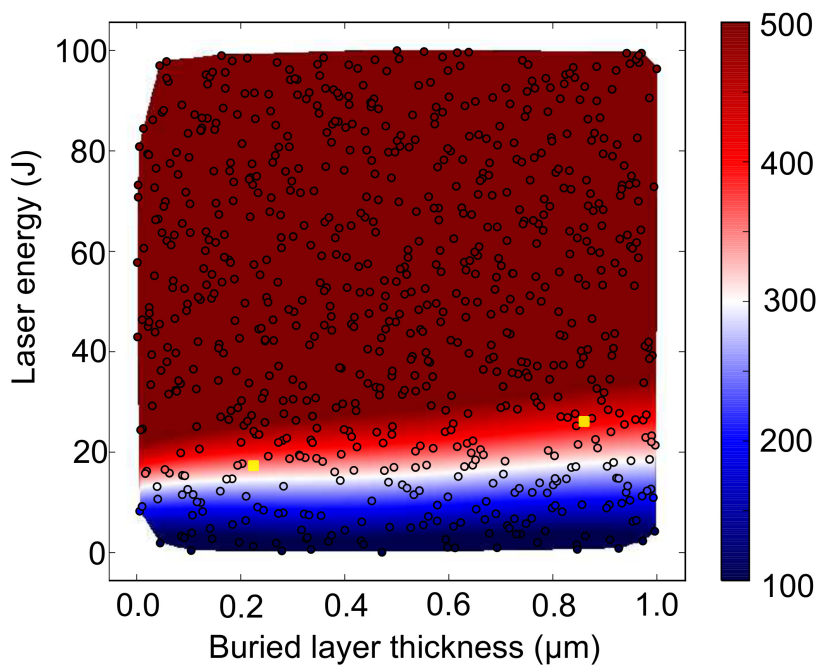

B

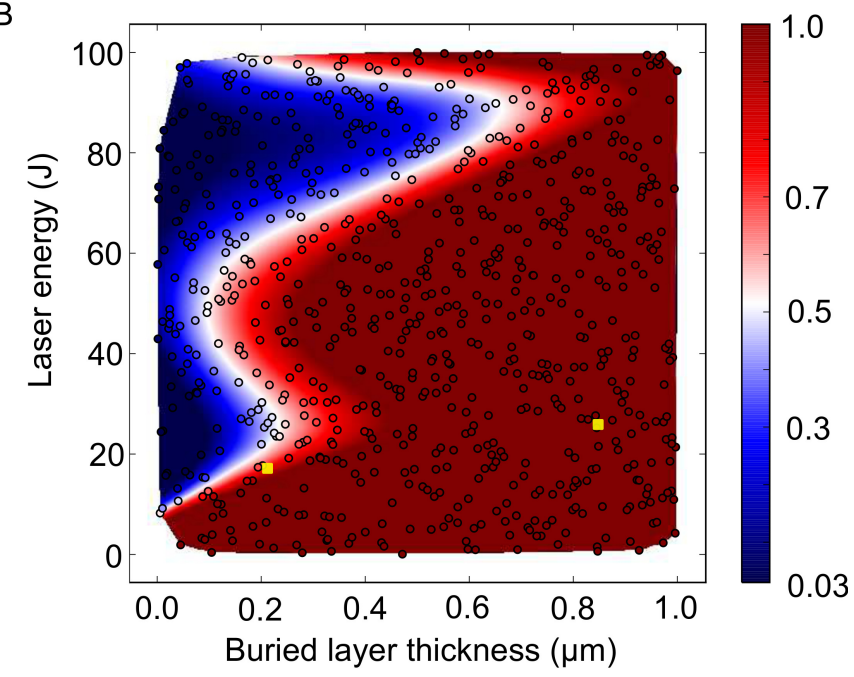

Figure 4: Two metric surrogates for the $300 \mathrm{eV}$ design case. The circles mark the design parameter values of the ensemble simulations. The yellow squares mark the design parameter values of the local minima. A) Peak temperature (eV). The color scale is limited to $100-500$ eV. B) Maximum optical depth over Fe emission range. The color scale is limited to 0.03 - 1.0.

buried layer. For the 200, 300, and $400 \mathrm{eV}$ cases, both minima failed to satisfy the temperature constraints because they reached temperatures that were too high. The thicker buried layer minima violated the temperature constraint more severely than the thinner minima. The minima do not satisfy the temperature constraints because the sulfur constraints (ratios and signals) are poorly met for low temperatures, therefore optimizing the cost function favors higher temperatures. For goal temperatures of $500 \mathrm{eV}$ and larger, the sulfur constraints are met and the optimization found minima that met the temperature constraints. For each design case, we compared the design constraints and cost function values of each minimum. We select the design with either the smallest cost function value, or, in the case of very similar cost function values, the design with the smallest optical depth as the optimal design.

The optimal designs and how well they met each design constraint are described in Table 6. In general, the relative difference between inferred opacity and model opacity ranges from $10-40 \%$. The lower temperature cases yielded optimized designs with temperatures larger than the goal temperature, as described above, because the sulfur dopant constraints push the optimization of the cost function toward higher temperatures. None of the optimized designs met the desired time period of constant temperature and density. In order to increase that period, we would need to increase the laser pulse length (for consideration of departure from LTE for longer pulse lengths see Section 4). The variation in density was higher than the desired $10 \%$, but was still less than the uncertainty in some densities inferred from K-shell line widths in existing experiments [8, 9, 57]. Although some of the designs had optical depths outside the desired range (0.03 to 0.64$)$, they were still less than 1 , therefore optical depth effects do 
not compromise the design much. The iron emission signal was sufficient for all optimized designs, but the dopant (ratios and signals) were only satisfied for the 750 and 1000 $\mathrm{eV}$ designs.

The fact that the sulfur line ratios and emission signals were only satisfied for the higher temperature designs suggests that sulfur is not a good dopant for lower temperatures. Two options for alternative dopant materials are magnesium and aluminum. Magnesium $\mathrm{H}-$ and $\mathrm{He}-$ like emission ranges from $1.25-1.8 \mathrm{keV}$, while aluminum emission ranges from $1.57-2.1 \mathrm{keV}$. Dopant emission that overlaps the iron emission range would complicate analysis of the dopant line ratios. The $200 \mathrm{eV}$ design has only small Fe emission in the $\mathrm{Mg}$ spectral range, allowing $\mathrm{Mg} \mathrm{K}$-shell emission to be a good dopant. The $300 \mathrm{eV}$ and $400 \mathrm{eV}$ designs have Fe emission from 0.76 - $1.5 \mathrm{keV}$, which overlaps $\mathrm{Mg} \mathrm{K}$-shell emission but not $\mathrm{Al} \mathrm{K-shell} \mathrm{emission.} \mathrm{The}$ overlap of the Fe emission with $\mathrm{Mg}$ emission is shown in Figure 5. The emission from 1.25 - $1.5 \mathrm{keV}$ would have a component coming from the Fe, making plasma temperature and density inference from the $\mathrm{Mg} \mathrm{K}$-shell lines more difficult. For the $300 \mathrm{eV}$ and $400 \mathrm{eV}$ designs, an Al dopant would be more appropriate since there is no overlap between the $\mathrm{Fe}$ and $\mathrm{Al}$ emission.

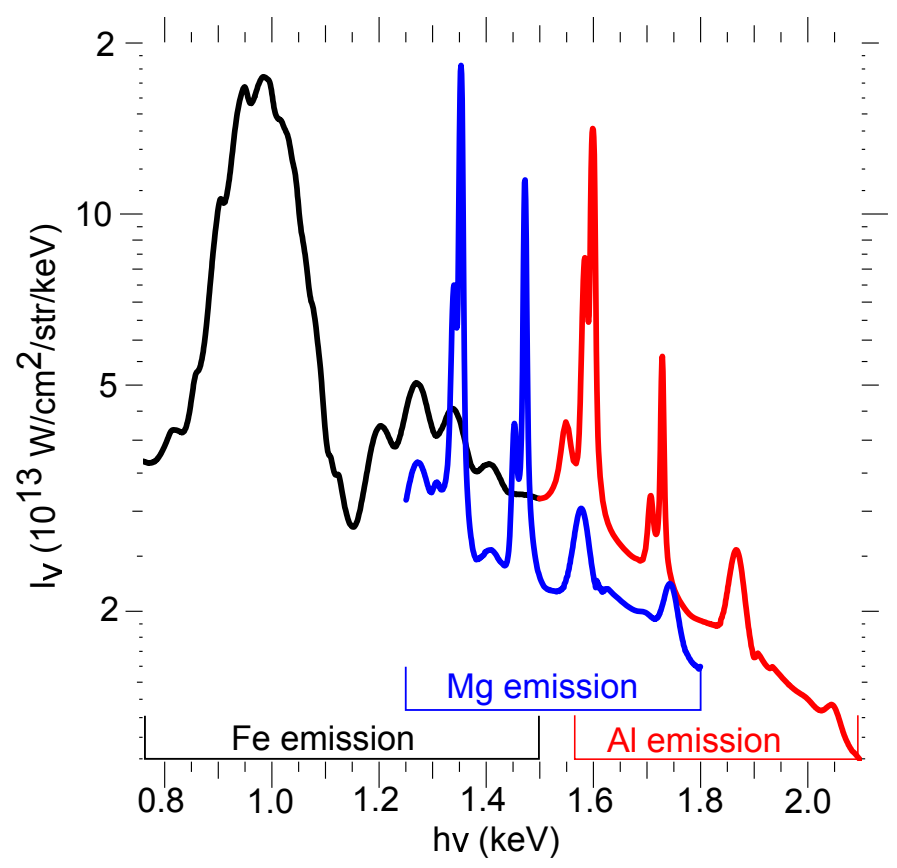

Figure 5: Simulated x-ray emission, at the time of peak electron temperature, at normal incidence from the target for the $300 \mathrm{eV}$ design. The black indicates the Fe emission, the red indicates the $\mathrm{Al}$ emission, and the blue indicates the $\mathrm{Mg}$ emission.

We executed HYDRA simulations with the S dopant replaced by either $\mathrm{Al}$ or $\mathrm{Mg}$ for each of the lower temperature designs. We used mixtures of $50 \% \mathrm{Fe}$ and $50 \% \mathrm{Mg}$ by atom and $58.5 \% \mathrm{Fe}$ and $41.5 \% \mathrm{Al}$ by atom. The initial material density of the mixed layers is calculated by

$$
\rho_{\mathrm{BL}}=\frac{1}{\frac{w_{\mathrm{Fe}}}{\rho_{\mathrm{Fe}}}+\frac{w_{\text {dopant }}}{\rho_{\text {dopant }}}}
$$

where $w_{\mathrm{Fe}}$ and $w_{\text {dopant }}$ are the mass fractions of $\mathrm{Fe}$ and dopant material in the buried layer. Using the results from the HYDRA simulations, we calculated the cost function, constraints, and single value metrics. The revised optimal designs and how well they met each design constraint are described in Table 7. Overall, these revised designs met the dopant ratio and line emission constraints. Although, they had slightly increased optical depths, the optical depths are still less than 1, which yield reasonable propagated uncertainties in the inferred opacity. We expect that if the ensemble was repeated for iron-magnesium and iron-aluminum buried layers, the optimization analysis would yield better designs, particularly at low temperatures $(200-400 \mathrm{eV})$.

The inferred and model opacity for the optimal $300 \mathrm{eV}$ and $750 \mathrm{eV}$ designs are shown in Figure 6. The figures are limited to the appropriate iron emission range for each design. Both designs have decent agreement between the inferred and model opacity $\left(\sigma_{\kappa_{\nu}}^{\text {rel }}<26 \%\right)$. For the 300 $\mathrm{eV}$ design, the difference between the inferred and model opacity for $0.76-0.86 \mathrm{keV}$ is due to tamper emission. The under-prediction of the inferred opacity from $0.92-1.2 \mathrm{keV}$ is an optical depth effect. The optical depth over 0.92 $1.2 \mathrm{keV}$ is larger than 0.7 but less than 1 , indicating that the opacity inference for this design is fairly sensitive to optical depth. The under-prediction occurs because the temperature used to infer the opacity (the average layer temperature), is higher than the temperature which contributes most to the x-ray emission for $0.92-1.2 \mathrm{keV}$ (a temperature occurring closer to the surface of the layer). For the $750 \mathrm{eV}$ design, the difference in the opacity valleys is due to the tamper emission. There is a small underprediction of the inferred opacity at the opacity peaks from $1.05-1.15 \mathrm{keV}$, but the difference in the opacity valleys contributes more to the average relative difference than the under-prediction.

We examined the propagated experimental uncertainties assuming a $10 \%$ uncertainty in the measured x-ray emission $\left(I_{\nu}\right)$, a $5 \%$ uncertainty in the temperature $(T)$, and a $10 \%$ relative uncertainty in the areal density $(\rho \Delta l)$ using the following equation:

$$
\begin{aligned}
\sigma_{\kappa_{\nu}}^{2}=\left(\frac{\partial \kappa_{\nu}}{\partial I_{\nu}}\right)^{2} \sigma_{I_{\nu}}^{2}+\left(\frac{\partial \kappa_{\nu}}{\partial B_{\nu}}\right)^{2} & \left(\frac{\partial B_{\nu}}{\partial T}\right)^{2} \sigma_{T}^{2} \\
& +\left(\frac{\partial \kappa_{\nu}}{\partial \rho \Delta l}\right)^{2} \sigma_{\rho \Delta l}^{2} .
\end{aligned}
$$

This analysis assumes there are no correlations between the uncertainties in the intensity measurements, inferred electron temperature, and areal density. These assumptions may not be accurate, so how best to address and 
A

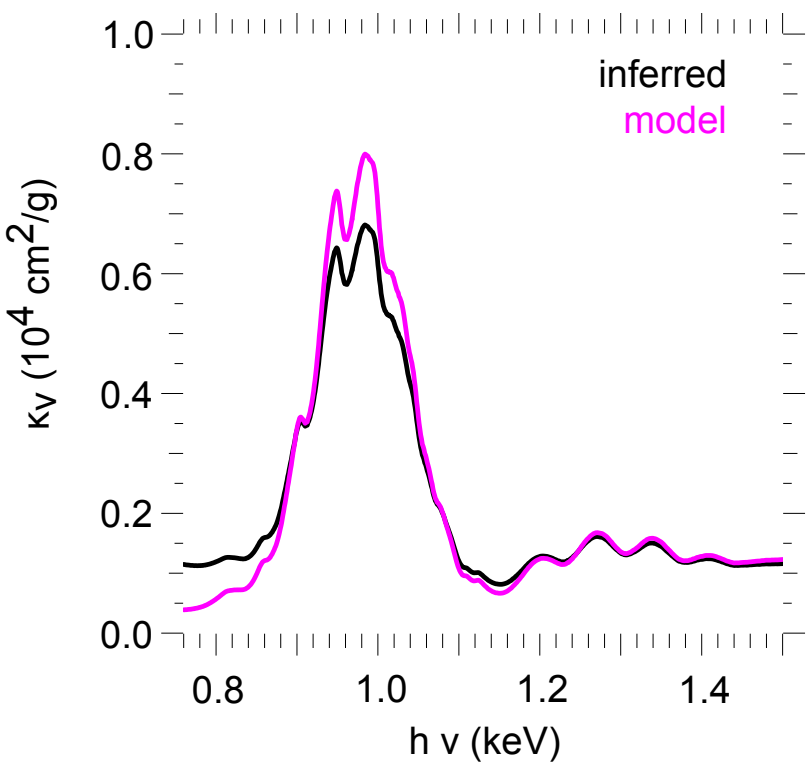

B

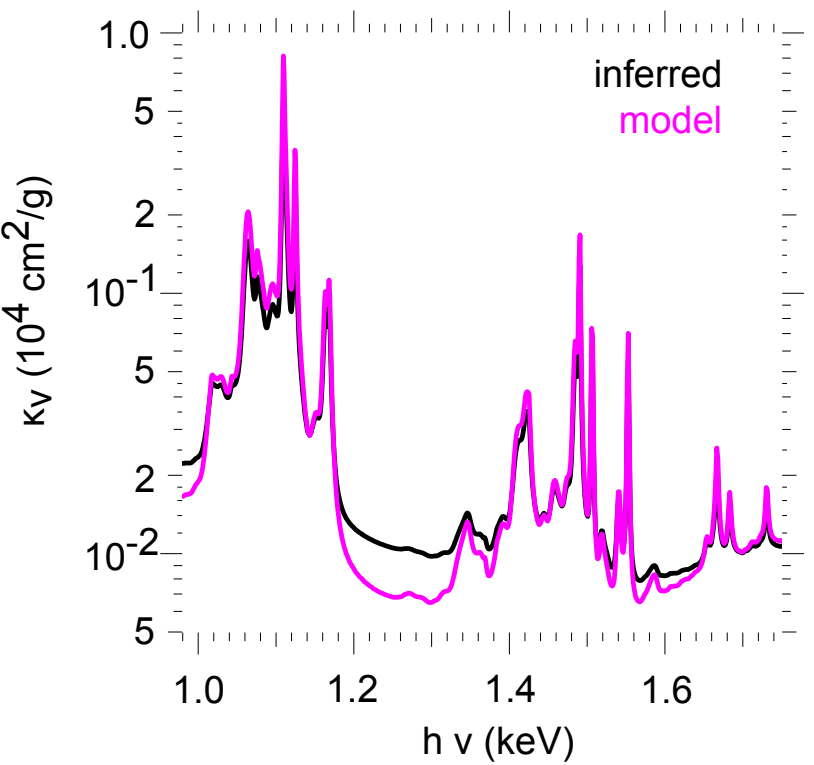

Figure 6: Inferred and model opacity over the appropriate iron emission range for two optimized designs. A) 300 eV design. B) 750 eV design, on a log-linear scale.

quantify the propagation of correlated experimental uncertainties should be examined in future work. For both designs, the propagated experimental uncertainties were less than $30 \%$ across the appropriate iron emission range. Previous work 33 indicated that propagated uncertainties can get very large for designs with large optical depths and a $30 \%$ propagated uncertainty is reasonable. Overall, these designs provide a good trade-off between minimizing optical depth and tamper emission effects while reaching the goal plasma conditions and ensuring adequate iron and dopant signals.

\subsection{Three Parameter Ensemble}

In order to more completely examine the design parameter space, we explored variations in the tamper thickness. We created ensembles varying laser energy $(E)$ from 0.1 to $100 \mathrm{~J}, \mathrm{FeS}_{2}$ buried layer thickness $\left(\Delta l_{\mathrm{BL}}\right)$ from $10 \mathrm{~nm}$ to 1 $\mu \mathrm{m}$, and tamper thickness $\left(\Delta l_{\mathrm{CH}}\right)$ from $10 \mathrm{~nm}$ to $10 \mu \mathrm{m}$. We fixed the laser pulse length at $0.5 \mathrm{ps}$. The parameters of the 7629 ensemble simulations were selected by Latin Hypercube sampling. We analyzed the ensemble for the same design cases as the two parameter ensemble ( $\mathrm{Ta}-$ ble 3. The design constraints, penalty parameters, and relative difference coefficient are the same as in the two parameter ensemble analysis (Section 3.2), except for the $1000 \mathrm{eV}$ design case. The $1000 \mathrm{eV}$ design cost function used a value of 1000 for the temperature constraints' (no. $1 \& 2$ ) penalty parameters in order focus the design on achieving that temperature.

\subsubsection{Surrogate quality}

Using the entire ensemble for training and testing yielded poor quality surrogates due to the rapid increase in the cost function values near some edges of the parameter space. The edges with large optical depths $(\tau>5)$ and high temperatures $\left(T^{\text {peak }}>1.9 \mathrm{keV}\right)$ yielded high cost function values $(>3000)$. Since optimized designs would not be found in those regions we removed them from the analysis. The number of simulations used to train and test each design case surrogate are included in Table 8 . We included more simulations to train the 750 and 1000 $\mathrm{eV}$ design case surrogates to ensure that optimal designs satisfied the temperature constraints and to improve the surrogate quality. A summary of the surrogate quality metrics for each design case is included in Table 8 .

\subsubsection{Optimized designs}

For each design case, the cost function and cost function surrogate appear very similar over the entire parameter space. However, for some local areas of the parameter space the surrogate either over-predicts or under-predicts the cost function. We are most concerned with how the cost function surrogate predicts the regions where the local minima are found. For each design case, we compared the cost function and surrogate values for the local minima. Similar results to those described below for the 300 $\mathrm{eV}$ case, were found for all design cases.

For each design case, the brute force method found one minimum and the basin hopping method found between 2 and 4 minima. The four minima found by brute force and basin hopping for the $300 \mathrm{eV}$ design case are described in Table 9. The cost function surrogate under-predicts all four local minima, but it under-predicts minimum 1 the most. The surrogate suggests that minimum 1 has the lowest value, but the actual cost function for minimum 2 is smaller. In addition, minimum 1 is a non-physical design because its temperature is less than $20 \mathrm{eV}$ making it a 
poor choice for all cases. The possibility for such underprediction (or potentially over-prediction) of the true cost function by the surrogate demonstrates the need to execute and analyze HYDRA simulations for each minimum before assessing the quality of the design.

We have compared two-dimensional plots for nine different fixed design parameters to understand the relationship between the the four local minima. The plots for the $300 \mathrm{eV}$ design case are presented in Figure 7. We have only plotted ranges of each parameter for which we had ensemble data. We have included each minimum, labeled by number, on the $2 \mathrm{D}$ slice that most closely matches its parameter values. The low surrogate value region in Figure $7 \mathrm{~A}$ without a square is connected to the low surrogate value region of Figure $7 \mathrm{~B}$ in which minimum 2 was found. Similarly, the low surrogate value region in Figure 7B without a square is connected to the low surrogate value region in Figure $7 \mathrm{~A}$ containing minimum 1. Similar statements could be made for Figure $7 \mathrm{D}$, Figure $7 \mathrm{E}$ and Figure $7 \mathrm{~F}$ Figure $7 \mathrm{H}$ indicates that minimum 1 was found in a region outside of where we had ensemble data, which may explain why the surrogate grossly under-predicted the cost function for that minimum. The $2 \mathrm{D}$ plots provide insight into how the regions in which local minima were found change as each parameter varies.

For each local minimum, we executed a HYDRA simulation with the appropriate design parameters. We calculated the single value metrics, constraints, and cost function using the HYDRA results. We selected the design with the smallest cost function value as the optimal design. As in the two parameter ensemble analysis, the optimized designs for the 200 - $400 \mathrm{eV}$ design cases did not meet the sulfur emission or ratio constraints, indicating a different dopant material is required. We replaced the sulfur dopant with an appropriate dopant material for each goal temperature. The designs with appropriate dopant materials and how well they met each design constraint are described in Table 10. The $400-1000 \mathrm{eV}$ designs meet all dopant emission and ratio constraints. The 200 and $300 \mathrm{eV}$ designs do not meet the $\mathrm{H}$ - and He-like $\alpha$ ratio constraint because the $\mathrm{H}-\alpha$ line emission is small. The $\mathrm{H}-\alpha$ line emission could be increased by including more of the dopant material in the buried layer which would also decrease the iron emission since there would be less iron in the buried layer. Including the concentration of dopant material as an additional design parameter should be explored in future work. An alternative way to increase dopant signal is to increase laser energy so the dopant material is heated to higher temperatures. In Section 3.2, the $200 \mathrm{eV}$ and 300 $\mathrm{eV}$ designs met dopant emission and ratio constraints but reached higher peak temperatures $\left(T^{\text {peak }} \approx 290\right.$ and 350 $\mathrm{eV}$, respectively). Determining the adequate temperature to meet dopant emission and ratio constraints should be addressed, in future work, by repeating the analysis for iron-magnesium and iron-aluminum buried layers.

The inferred and model opacity for the optimal $300 \mathrm{eV}$ and $750 \mathrm{eV}$ designs, determined from the three parameter ensembles, are shown in Figure 8. Over the iron emission ranges, both designs have good agreement between the inferred and model opacity $\left(\sigma_{\kappa_{\nu}}^{\text {rel }}<21 \%\right)$. For the $300 \mathrm{eV}$ design, the differences between the inferred and model opacity are predominantly due to tamper emission. For the $750 \mathrm{eV}$ design, the differences in the opacity valleys $(0.95-1.0 \mathrm{keV}$ and $1.18-1.38 \mathrm{keV})$ are due to the tamper emission. For both designs, the propagated experimental uncertainties, assuming a $10 \%$ uncertainty in the measured x-ray emission, a $5 \%$ uncertainty in the temperature, and a $10 \%$ uncertainty in the areal density, were less than $35 \%$ across the iron emission range. Overall, these designs reach plasma condition goals and provide adequate iron and dopant signals while minimizing optical depth and tamper emission effects.

There are some commonalities between the designs found by the two and three parameter ensembles. The 200 - $750 \mathrm{eV}$ optimal designs found in both ensembles have a small range of buried layer thicknesses between 0.17 and $0.28 \mu \mathrm{m}$. The $1000 \mathrm{eV}$ designs have thicker buried layers $(0.4-0.6 \mu \mathrm{m})$. For all design cases, except the $200 \mathrm{eV}$ design, the optimal laser energies were similar in both the two and three parameter ensembles. The $200 \mathrm{eV}$ design found in the three parameter ensemble requires more energy than that of the design found in the two parameter ensemble because its tamper thicknesses are much larger. For all other cases, the optimized tamper thicknesses ranged from 2.4 $3 \mu \mathrm{m}$, suggesting that the assumed tamper thickness of 3 $\mu \mathrm{m}$ in the two parameter ensemble was reasonable. Overall, both ensembles suggest a range of laser energies (12 - $60 \mathrm{~J}$ ) incident on $0.2-0.3 \mu \mathrm{m}$ buried layers surrounded by $2-3 \mu \mathrm{m}$ tampers would access to plasma conditions consistent with the radiative zone of the sun (200 - 1000 $\mathrm{eV}$ and $\rho \approx 4.2 \mathrm{~g} / \mathrm{cm}^{3}$ ).

All designs yield very small periods of constant plasma conditions $(0.2-0.4 \mathrm{ps})$. These periods are less than the temporal resolution of previous experiments $(\approx 1$ $2 \mathrm{ps}$ ), meaning that the measured emission would capture a range of plasma conditions. We suggest two ways to increase the period of constant plasma conditions. The first is increasing laser pulse length, which elongates the duration of constant plasma conditions at the time of peak electron temperature 33]. For consideration of departure from LTE for longer pulse lengths see Section 4. The second, is to focus the design optimization on a time, after buried layer recompression, during which the density is relatively constant. The duration of constant recompressed density decreases with increasing laser pulse and increases with increasing tamper thickness [33. A design optimization focusing on aligning periods of constant temperature with the period of constant recompressed density could be created. This type of design optimization would focus on a time when the target is cooling, which will complicate the ability to reach goal temperatures. Nonetheless, our process could be applied to such an approach. Future work should include the application of the automated design process to a variable laser energy, laser pulse length, buried 
A

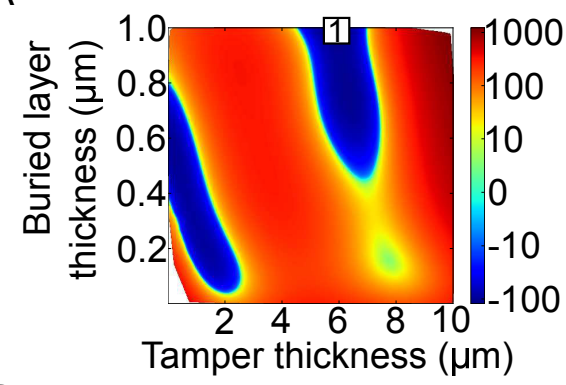

D

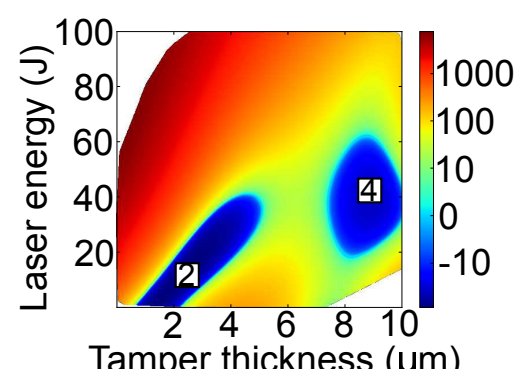

G

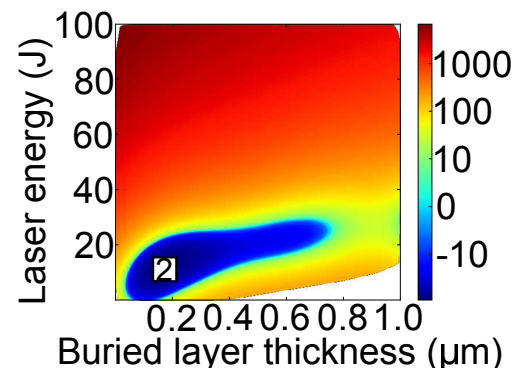

B

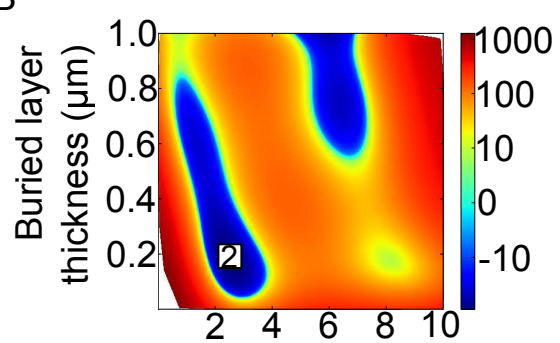

Tamper thickness $(\mu \mathrm{m})$

E

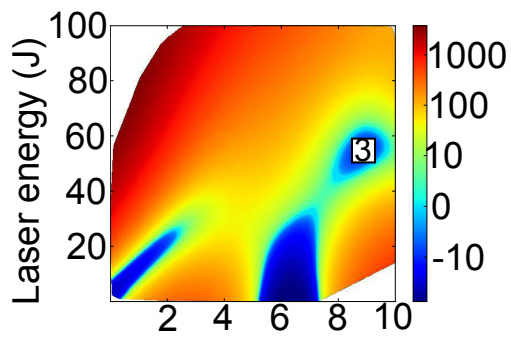

Tamper thickness $(\mu \mathrm{m})$

$\mathrm{H}$

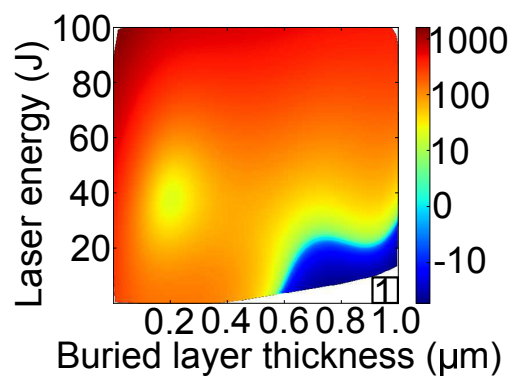

C

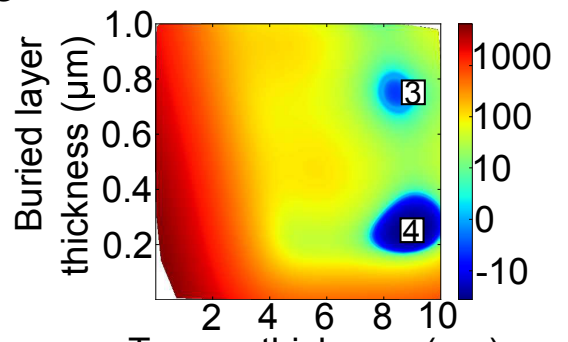

Tamper thickness $(\mu \mathrm{m})$

F

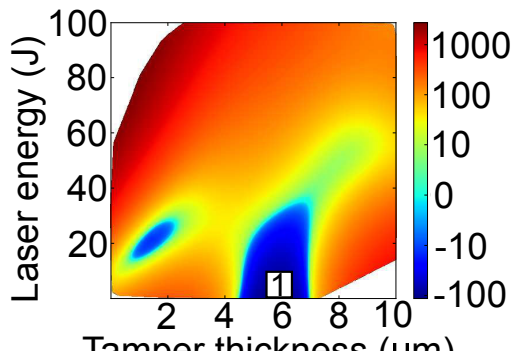

Tamper thickness $(\mu \mathrm{m})$

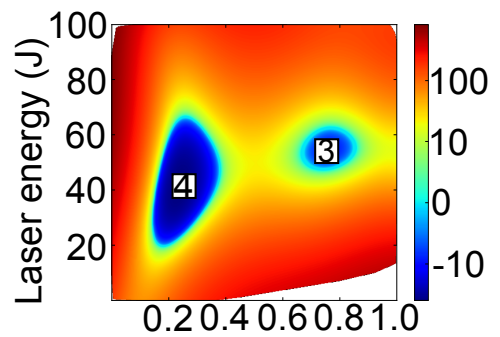

Buried layer thickness $(\mu \mathrm{m})$

Figure 7: Two dimensional plots for the $300 \mathrm{eV}$ design cost function surrogate. The white squares mark the local minima identified by the optimization methods. The numbers, as listed in Table 9 mark the local minima. A) At 0.1 J. B) At 13 J. C) At 48 J. D.) At $0.22 \mu$ m buried layer thickness. E) At $0.76 \mu \mathrm{m}$ buried layer thickness. F) At $1.0 \mu \mathrm{m}$ buried layer thickness. G) At $2.5 \mu \mathrm{m}$ tamper thickness. H) At $5.8 \mu \mathrm{m}$ tamper thickness. I) At $8.8 \mu \mathrm{m}$ tamper thickness.

layer thickness, and tamper thickness ensembles aimed at optimization at the time of peak electron temperature and at the time of constant recompressed density.

\section{Discussion and Conclusions}

This work explores the computational design of short pulse laser driven iron opacity experiments. In such experiments, target materials can be heated to several hundreds of eVs while maintaining densities $1 / 10$ of solid to solid density. This enables access to conditions relevant to the upper radiative zone of the sun $(200-400 \mathrm{eV}$ and densities larger than $1 / 10$ of solid density). It also enables access to higher temperatures consistent with the deep radiative zone of the sun (up to $1000 \mathrm{eV}$ ), however, at lower electron densities. Spectroscopic measurements at temperatures of $200-1000 \mathrm{eV}$ and densities of $1 / 10$ of solid to solid density would investigate the validity of theoretical opacity models at stellar-relevant conditions. Such measurements may aid in resolving the current disagreement between solar parameters calculated from model and observations.
We developed an automated design process using Lawrence Livermore National Laboratory's (LLNL) Uncertainty Quantification Pipeline (UQP) to find globally optimized laser and target parameters that satisfy a variety of design goals. We used the automated design process to find optimized designs for single and multiple parameter ensembles of $\mathrm{FeS}_{2}$ buried layer targets. We explored a set of design cases relevant to the upper radiative zone of the sun: $T=200$ to $400 \mathrm{eV}$ and $\rho>1 / 10$ of solid density. In addition, we explored a set of design cases for higher temperatures at a constant density: $T=500$ to $1000 \mathrm{eV}$ and $\rho=4.2 \mathrm{~g} / \mathrm{cm}^{3}$. Ensembles individually varying laser energy, laser pulse length, buried layer thickness, and tamper thicknesses demonstrated that the cost function is most sensitive to the goal temperature and optical depth constraints. The laser energy ensemble suggested that, in order to reach temperatures of $200-1000 \mathrm{eV}$, laser energies of 10 - $50 \mathrm{~J}$, respectively are needed. We explored two parameter designs, varying laser energy and buried layer thickness, as well as three parameter designs, varying laser energy, buried layer thickness, and tamper thickness. 
A

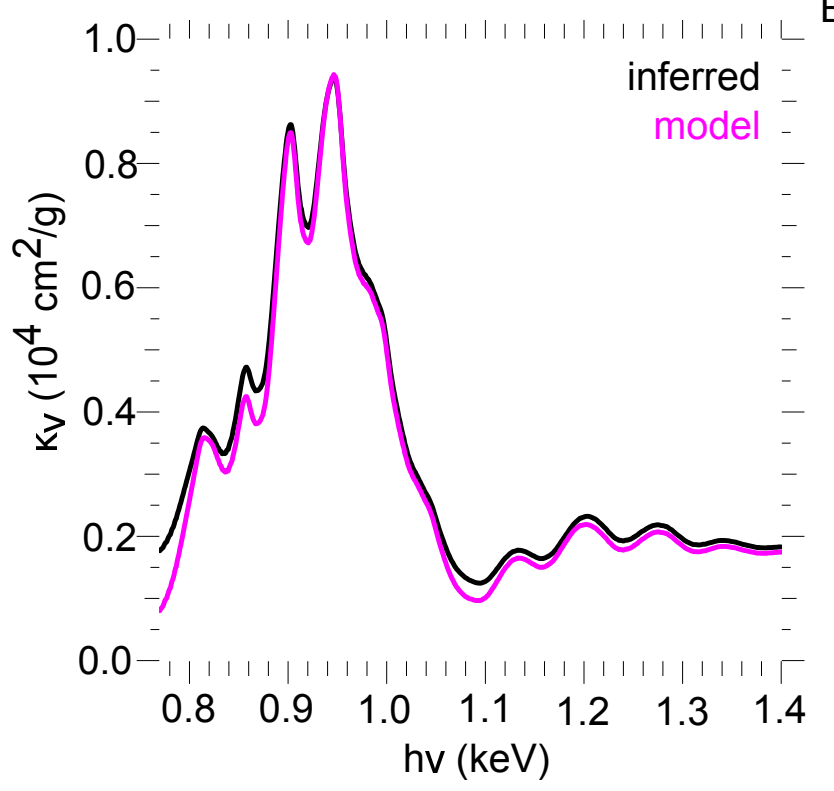

B

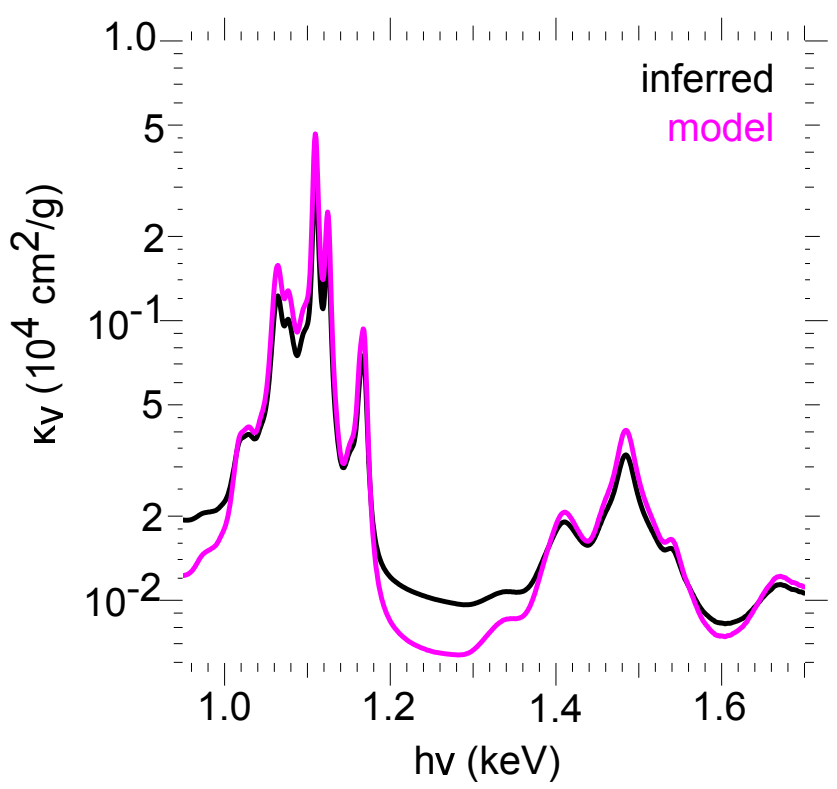

Figure 8: Inferred and model opacity over the appropriate iron emission range for two optimized designs determined from the analysis of the three parameter ensemble. A) $300 \mathrm{eV}$ design. B) $750 \mathrm{eV}$ design, on a log-linear scale.

From the analysis of these ensembles suggest that different dopant materials are required for different temperature goals: magnesium for up to $300 \mathrm{eV}$, aluminum for 300 to $500 \mathrm{eV}$, and sulfur for larger than $500 \mathrm{eV}$. For all optimized designs, the average relative difference between the inferred and model opacity was reasonable $(<31 \%)$. However, the time period of constant plasma conditions $(0.2-$ $0.4 \mathrm{ps}$ ) was significantly smaller than the temporal resolution of previous experiments $(1-2 \mathrm{ps})$. Within the design optimization outlined in this paper, the period of constant plasma conditions could be increased by increasing laser pulse length. Alternatively, a design optimization focused on a time during constant recompressed density could be completed to maximize the duration of constant plasma conditions.

We have suggested that increasing laser pulse length would increase the period of constant plasma conditions. Increasing pulse length will increase the period for which the target is heating. Previously, we found that, for $1 \mathrm{ps}$ pulses, the largest departures from LTE occur as the target is heating [33. We now consider a 5 ps pulse model that reaches $300 \mathrm{eV}$ and $2.4 \mathrm{~g} / \mathrm{cm}^{3}$ around $10 \mathrm{ps}$ to address how increasing pulse length may impact departures from LTE. Analysis of 5 ps pulses yield similar results as previously analyzed 1 ps pulses. The non-LTE effects on plasma conditions (small), opacities (moderate), and emissivities (moderate) are strongest while the target is heating (4 to $10 \mathrm{ps}$ ). However, we are more concerned with the non-LTE effects during the period of constant plasma conditions (8 to $12 \mathrm{ps}$ ) and peak emission ( 8 to $18 \mathrm{ps}$ ). Departures from LTE over the time of peak emission are moderate $(<10$ $\%$, which minimally affects the time-integrated emission $(<5 \%)$ and moderately affects of time resolved emission
$(<15 \%)$. The ability to distinguish these non-LTE effects depends on the time resolution and uncertainty in experimental measurements. We expect as the pulse length is increased at similar peak temperatures (up to $300 \mathrm{eV}$ ), there may be concerns with non-LTE effects during heating of the target but for the period when plasma temperature and emission are largest, we expect non-LTE effects on experimental observables to be small to moderate.

All of the simulations assume a simple parametric energy source to account for the absorption of laser energy, the conversion of absorbed energy into hot electrons, and the deposition of hot electron energy into the target materials. Using our assumed energy source to model previous experiments has been shown to predict peak plasma conditions and reasonable $\mathrm{x}$-ray pulse durations. However, we recognize that our methodology does not address the experimental evidence suggesting that heating may be longer than the laser pulse length [58,62 and that hot electron heating laterally spreads outside of the laser focal spot size. In addition, our 1D modeling does not include experimental details, which may cause $2 \mathrm{D}$ effects, such as roughness of layers, spatial speckling of the laser spot, and spatial inhomogeneities in mixed buried layers. Also, all mixed buried layers in this paper assume an atom mixture between two materials, which may not be physically achievable. In such a case, it is common for the buried layer to be made of a series of alternating layers between the desired materials. Our methodology could be applied to such a target by including the individual layers into the model.

While we believe that our methodology is useful for designing experiments, more detailed modeling would likely be needed to analyze experimental results. Specifically, 
more detailed modeling that better captures the temporal and spatial dependence of short pulse laser heating mechanisms may be necessary. One idea is to couple the results of a high resolution particle-in-cell simulation of the short pulse laser absorption with a hot electron transport simulation that includes the recirculation of hot electrons, then create an interface between the hot electron transport simulation and a radiation hydrodynamic simulation such that heating source is informed by the hot electron transport. A similar method has been used by Hoarty et al. to analyze streaked K-shell emission 35]. Such a coupled calculation requires substantial resources to develop, complete, and analyze, limiting its use as a relatively quick design tool. We developed a computational design process that allows for the relatively rapid determination of optimized designs given some design goal and constraints.

\section{Acknowledgments}

The authors thank S. Brandon and D. Domyancic for support with using the UQP for surrogate based optimization; R. Nora, L. Peterson, and B. Spears for discussions on using the UQP for managing and analyzing ensembles of HYDRA simulations. This work was performed under the auspices of the U.S. Department of Energy by Lawrence Livermore National Laboratory under Contract DE-AC5207NA27344. Lawrence Livermore National Security, LLC.

[1] K. Nazir, S. J. Rose, A. Djaoui, G. J. Tallents, M. G. Holden, P. A. Norreys, P. Fews, J. Zhang, F. Failles, Xray spectroscopic studies of hot, dense iron plasma formed by subpicosecond high intensity KrF laser irradiation Appl. Phys. Let. 69 (24) (1996) 3686-3688. doi:10.1063/1.117189 URL http://dx.doi.org/10.1063/1.117189

[2] K. Eidmann, U. Andiel, F. Pisani, P. Hakel, R. C. Mancini, G. C. Junkel-Vives, J. Abdallah, K. Witte, K-shell spectra from hot dense aluminum layers buried in carbon and heated by ultrashort laser pulses J. of Quant. Spectrosc. and Radiat. Transf. $81(1-4)(2003) 133-146$. URL http://dx.doi .org/10.1016/S0022-4073(03)00067-0

[3] P. Audebert, P. Renaudin, S. Bastiani-Ceccotti, J.-P. Geindre, C. Chenais-Popovics, S. Tzortzakis, V. Nagels-Silvert, R. Shepherd, I. Matsushima, S. Gary, F. Girard, O. Peyrusse, J.C. Gauthier, Picosecond time-resolved x-ray absorption spectroscopy of ultrafast aluminum plasmas, Phys. Rev. Lett. 94 (2005) 025004.

URL http://dx.doi.org/10.1103/PhysRevLett.94.025004

[4] D. J. Hoarty, S. F. James, H. Davies, C. R. D. Brown, J. W. O. Harris, C. C. Smith, S. J. Davidson, E. Kerswill, B. J. B. Crowley, S. J. Rose, Heating of buried layer targets by $1 \omega$ and $2 \omega$ pulses using the HELEN CPA laser High Energy Density Phys. $3(1-2)(2007) 115-119$.

URL http://dx.doi .org/10.1016/j.hedp.2007.02.007

[5] D. J. Hoarty, S. F. James, C. R. D. Brown, B. M. Williams, T. Guymer, M. Hill, J. Morton, D. Chapman, R. Shepherd, J. Dunn, G. Brown, M. Schneider, P. Beiersdorfer, H. K. Chung, J. W. O. Harris, L. Upcraft, C. C. Smith, R. W. Lee, High temperature, high density opacity measurements using short pulse lasers $\mid$ J. of Phys.: Conf. Ser. 244 (1) (2010) 012002. URL http://dx.doi.org/10.1088/1742-6596/244/1/012002

[6] D. J. Hoarty, S. F. James, C. R. D. Brown, B. M. Williams, H. K. Chung, J. W. O. Harris, L. Upcraft, B. J. B. Crowley, C. C. Smith, R. W. Lee, Measurements of emission spectra from hot, dense germanium plasma in short pulse laser experiments, High Energy Density Phys. 6 (1) (2010) 105 - 108. doi:10. 1016/j.hedp. 2009.05.019

URL http://dx.doi.org/10.1016/j.hedp.2009.05.019
[7] C. R. D. Brown, D. J. Hoarty, S. F. James, D. Swatton, S. J. Hughes, J. W. Morton, T. M. Guymer, M. P. Hill, D. A. Chapman, J. E. Andrew, A. J. Comley, R. Shepherd, J. Dunn, H. Chen, M. Schneider, G. Brown, P. Beiersdorfer, J. Emig, Measurements of electron transport in foils irradiated with a picosecond time scale laser pulse, Phys. Rev. Lett. 106 (2011) 185003.

URL http://dx.doi.org/10.1103/PhysRevLett.106.185003

[8] D. J. Hoarty, P. Allan, S. F. James, C. R. D. Brown, L. M. R. Hobbs, M. P. Hill, J. W. O. Harris, J. Morton, M. G. Brookes, R. Shepherd, J. Dunn, H. Chen, E. Von Marley, P. Beiersdorfer, H. K. Chung, R. W. Lee, G. Brown, J. Emig, Observations of the effect of ionization-potential depression in hot dense plasma Phys. Rev. Lett. 110 (2013) 265003. doi:10.1103/PhysRevLett. 110.265003

URL http://dx.doi.org/10.1103/PhysRevLett.110.265003

[9] D. J. Hoarty, P. Allan, S. F. James, C. R. D. Brown, L. M. R. Hobbs, M. P. Hill, J. W. O. Harris, J. Morton, M. G. Brookes, R. Shepherd, J. Dunn, H. Chen, E. Von Marley, P. Beiersdorfer, H. K. Chung, R. W. Lee, G. Brown, J. Emig, The first data from the orion laser; measurements of the spectrum of hot, dense aluminium High Energy Density Phys. 9 (4) (2013) $661-671$. URL http://dx.doi.org/10.1016/j.hedp.2013.06.005

[10] B. K. F. Young, B. G. Wilson, D. F. Price, R. E. Stewart, Measurement of x-ray emission and thermal transport in nearsolid-density plasmas heated by 130 fs laser pulses Phys. Rev. E 58 (1998) 4929-4936. doi:10.1103/PhysRevE.58.4929. URL http://dx.doi.org/10.1103/PhysRevE.58.4929

[11] J. W. O. Harris, L. M. Upcraft, D. J. Hoarty, B. J. B. Crowley, C. R. D. Brown, S. F. James, A comparison of theory and experiment for high density, high temperature germanium spectra, High Energy Density Phys. 6 (1) (2010) $95-98$. URL http://dx.doi.org/10.1016/j.hedp.2009.06.001

[12] M. Shahzad, G. J. Tallents, A. B. Steel, L. Hobbs, D. J. Hoarty, J. Dunn, Electron temperature and density characterization using l-shell spectroscopy of laser irradiated buried iron layer targets, Phys. of Plasmas 21 (8) (2014) 082702. arXiv:http: //dx.doi.org/10.1063/1.4892263 doi:10.1063/1.4892263 URL http://dx.doi.org/10.1063/1.4892263

[13] J. N. Bahcall, M. H. Pinsonneault, What do we (not) know theoretically about solar neutrino fluxes? Phys. Rev. Lett. 92 (2004) 121301. doi:10.1103/PhysRevLett.92.121301 URL http://link.aps.org/doi/10.1103/PhysRevLett.92. 121301

[14] S. Turck-Chièze, S. Couvidat, L. Piau, J. Ferguson, P. Lambert, J. Ballot, R. A. García, P. Nghiem, Surprising sun: A new step towards a complete picture? Phys. Rev. Lett. 93 (2004) 211102. doi:10.1103/PhysRevLett.93.211102. URL http://link.aps.org/doi/10.1103/PhysRevLett.93. 211102

[15] S. Basu, H. M. Antia, Constraining solar abundances using helioseismology The Astrophys. J. Lett. 606 (1) (2004) L85. URL http://stacks. iop.org/1538-4357/606/i=1/a=L85

[16] J. N. Bahcall, S. Basu, M. Pinsonneault, A. M. Serenelli, Helioseismological implications of recent solar abundance determinations, The Astrophys. J. 618 (2) (2005) 1049. URL http://stacks . iop.org/0004-637X/618/i=2/a=1049

[17] J. N. Bahcall, A. M. Serenelli, S. Basu, New solar opacities, abundances, helioseismology, and neutrino fluxes The Astrophys. J. Lett. 621 (1) (2005) L85, see the standard solar model (BS2005-OP) at http://www.sns.ias.edu/ jnb/ URL http://stacks .iop.org/1538-4357/621/i=1/a=L85

[18] S. Turck-Chièze, F. Delahaye, D. Gilles, G. Loisel, L. Piau, On plasma radiative properties in stellar conditions High Energy Density Phys. 5 (3) (2009) $132-138$ URL http://dx.doi .org/10.1016/j.hedp.2009.06.007

[19] S. Basu, H. M. Antia, Helioseismology and solar abundances, Phys. Rep. $457(5-6)(2008) 217-283$. doi:10.1016/j. physrep.2007.12.002

URL http://www.sciencedirect.com/science/article/pii/ S0370157307004565 
[20] M. Asplund, N. Grevesse, A. J. Sauval, The solar chemical composition, in: F. N. Bash, T. G. Barnes (Eds.), Cosmic Abundances as Records of Stellar Evolution and Nucleosynthesis, ASP Conference Series, Astronomical Society of the Pacific, 2005, p. 25.

[21] M. Asplund, N. Grevesse, A. J. Sauval, The solar chemical composition, Nucl. Phys. A 777 (2006) 1 - 4 . doi:10.1016/ j.nuclphysa.2005.06.010

URL http://dx.doi.org/10.1016/j.nuclphysa.2005.06.010

[22] L. B. Da Silva, B. J. MacGowan, D. R. Kania, B. A. Hammel, C. A. Back, E. Hsieh, R. Doyas, C. A. Iglesias, F. J. Rogers, R. W. Lee, Absorption measurements demonstrating the importance of $\Delta n=0$ transitions in the opacity of iron, Phys. Rev. Lett. 69 (1992) 438-441. doi:10.1103/PhysRevLett.69.438 URL http://link.aps.org/doi/10.1103/PhysRevLett.69.438

[23] P. T. Springer, D. J. Fields, B. G. Wilson, J. K. Nash, W. H. Goldstein, C. A. Iglesias, F. J. Rogers, J. K. Swenson, M. H. Chen, A. Bar-Shalom, R. E. Stewart, Spectroscopic absorption measurements of an iron plasma Phys. Rev. Lett. 69 (1992) 3735-3738. URL http://dx.doi.org/10.1103/PhysRevLett.69.3735

[24] G. Winhart, K. Eidmann, C. A. Iglesias, A. Bar-Shalom, E. Minguez, A. Rickert, S. J. Rose, Xuv opacity measurements and comparison with models, Journal of Quantitative Spectroscopy and Radiative Transfer 54 (1) (1995) 437-446. URL http://dx.doi.org/10.1016/0022-4073(95)00080-5

[25] G. Winhart, K. Eidmann, C. A. Iglesias, A. Bar-Shalom, Measurements of extreme uv opacities in hot dense al, fe, and ho Phys. Rev. E 53 (1996) R1332-R1335. doi:10.1103/PhysRevE. 53.R1332

URL http://link. aps.org/doi/10.1103/PhysRevE.53.R1332

[26] C. Chenais-Popovics, H. Merdji, T. Missalla, F. Gilleron, J.C. Gauthier, T. Blenski, F. Perrot, M. Klapisch, C. BaucheArnoult, J. Bauche, A. Bachelier, K. Eidmann, Opacity studies of iron in the 15-30ev temperature range, The Astrophysical Journal Supplement Series 127 (2) (2000) 275. URL http: //stacks.iop.org/0067-0049/127/i=2/a=275

[27] G. Loisel, P. Arnault, S. Bastiani-Ceccotti, T. Blenski, T. Caillaud, J. Fariaut, W. Fölsner, F. Gilleron, J.-C. Pain, M. Poirier, C. Reverdin, V. Silvert, F. Thais, S. Turck-Chièze, B. Villette, Absorption spectroscopy of mid and neighboring $\mathrm{z}$ plasmas: Iron, nickel,copper and germanium High Energy Density Physics 5 (3) (2009) $173-181$ URL http://dx.doi.org/10.1016/j.hedp.2009.05.015

[28] T. Blenski, G. Loisel, M. Poirier, F. Thais, P. Arnault, T. Caillaud, J. Fariaut, F. Gilleron, J.-C. Pain, Q. Porcherot, C. Reverdin, V. Silvert, B. Villette, S. Bastiani-Ceccotti, S. Turck-Chièze, W. Foelsner, F. de Gaufridy de Dortan, Opacity of iron, nickel, and copper plasmas in the x-ray wavelength range: Theoretical interpretation of $2 p-3 d$ absorption spectra. Phys. Rev. E 84 (2011) 036407.

URL http://dx.doi.org/10.1103/PhysRevE.84.036407

[29] J. E. Bailey, G. A. Rochau, C. A. Iglesias, J. Abdallah, Jr., J. J. MacFarlane, I. Golovkin, P. Wang, R. C. Mancini, P. W. Lake, T. C. Moore, M. Bump, O. Garcia, S. Mazevet, Ironplasma transmission measurements at temperatures above 150 ev, Phys. Rev. Lett. 99 (2007) 265002.

URL http://dx.doi.org/10.1103/PhysRevLett.99.265002

[30] J. E. Bailey, G. A. Rochau, R. C. Mancini, C. A. Iglesias, J. J. MacFarlane, I. E. Golovkin, J. C. Pain, F. Gilleron, C. Blancard, P. Cosse, G. Faussurier, G. A. Chandler, T. J. Nash, D. S. Nielsen, P. W. Lake, Diagnosis of x-ray heated $\mathrm{Mg} / \mathrm{Fe}$ opacity research plasmas, Rev. of Sci. Instrum. 79 (11) (2008) 113104. arXiv:http://dx.doi.org/10.1063/1.3020710 doi:10.1063/1.3020710

URL http://dx.doi.org/10.1063/1.3020710

[31] J. E. Bailey, G. A. Rochau, R. C. Mancini, C. A. Iglesias, J. J. MacFarlane, I. E. Golovkin, C. Blancard, P. Cosse, G. Faussurier, Experimental investigation of opacity models for stellar interior, inertial fusion, and high energy density plasmas Phys. of Plasmas 16 (5) (2009) 058101. arXiv:http://dx.doi.org/
10.1063/1.3089604 doi:10.1063/1.3089604

URL http://dx.doi.org/10.1063/1.3089604

[32] J. E. Bailey, T. Nagayama, G. P. Loisel, G. A. Rochau, C. Blancard, J. Colgan, Ph. Cosse, G. Faussurier, C. J. Fontes, F. Gilleron, I. Golovkin, S. B. Hansen, C. A. Iglesias, D. P. Kilcrease, J. J. MacFarlane, R. C. Mancini, S. N. Nahar, C. Orban, J.-C. Pain, A. K. Pradhan, M. Sherrill, B. G. Wilson, A higher-than-predicted measurement of iron opacity at solar interior temperatures, Nat. 517 (2015) 56-59.

URL http://dx.doi.org/10.1038/nature14048

[33] M. E. Martin, R. A. London, S. Goluoglu, H. D. Whitley, Computational design of short pulse laser driven iron opacity experiments, Phys. of Plasmas 24 (2) (2017) 022705. URL http://dx.doi.org/10.1063/1.4976710

[34] Atomic Weapons Establishment, Laser performance, $\quad$ http://www.awe.co.uk/what-we-do/ science-engineering-technology/orion-laser-facility/ laser-performance/, 2017 (accessed 05.15.2017).

[35] D. J. Hoarty, N. Sircombe, P. Beiersdorfer, C. R. D. Brown, M. Hill, L. R. Hobbs, S. James, J. Morton, E. Hill, M. Jeffery, J. Harris, R. Shepherd, E. Marley, E. Magee, J. Emig, J. Nilsen, H. Chung, R. Lee, S. Rose, Modelling k shell spectra from short pulse heated buried microdot targets High Energy Density Physics 23 (Supplement C) (2017) 178 - 183. doi:https://doi.org/10.1016/j.hedp.2017.04.004 URL http://www.sciencedirect.com/science/article/pii/ S157418181730037X

[36] S. H. Langer, B. Spears, J. L. Peterson, J. E. Field, R. Nora, S. Brandon, A HYDRA UQ workflow for nif ignition experiments in: Proceedings of the 2nd Workshop on In Situ Infrastructures for Enabling Extreme-scale Analysis and Visualization, ISAV '16, IEEE Press, Piscataway, NJ, USA, 2016, pp. 1-6.

URL http://dl.acm.org/citation. cfm?id=3018860\&CFID= 941605421\&CFTOKEN=76422605

[37] D. D. Lucas, R. Klein, J. Tannahill, D. Ivanova, S. Brandon, D. Domyancic, Y. Zhang, Failure analysis of parameter-induced simulation crashes in climate models Geosci. Model Dev. 6 (4) (2013) 1157-1171. doi:10.5194/gmd-6-1157-2013 URL http://www.geosci-model-dev.net/6/1157/2013/

[38] S. Brandon, D. Domyancic, J. Tannahill, D. Lucas, G. Christianson, J. McEnerney, R. I. Klein, Ensemble calculations via the LLNL UQP Pipeline: A user's guide, Tech. Rep. LLNL-SM480999, Lawrence Livermore National Laboratory (2011).

[39] M. M. Marinak, G. D. Kerbel, N. A. Gentile, O. Jones, D. Munro, S. Pollaine, T. R. Dittrich, S. W. Haan, Threedimensional HYDRA simulations of National Ignition Facility targets, Phys. of Plasmas 8 (5) (2001) 2275-2280. doi: 10.1063/1.1356740

URL http://scitation.aip.org/content/aip/journal/pop/ 8/5/10.1063/1.1356740

[40] The SciPy community, SciPy is open-source software for mathematics, science, and engineering, https://docs.scipy.org/ doc/scipy/reference/index.html 2016 (accessed 05.15.2017).

[41] D. Munro, Yorick, http://yorick.sourceforge.net/, 2011 (accessed 05.15.2017).

[42] E. K. P. Chong, S. H. Żak, An Introduction to Optimization, 3rd Edition, Wiley \& Sons, New Jersey, 2008.

[43] J. Sobieszczanski-Sobieski, A. Morris, M. J. L. van Tooren, G. L. Rocca, W. Yao, Multidisciplinary Design Optimization Supported by Knowledge Based Engineering, Wiley \& Sons, United Kingdom, 2015.

[44] M. E. Martin, Computational design of short pulse laser driven iron opacity measurements at stellar-relevant conditions, Ph.D. thesis (University of Florida, Gainesville, 2017).

[45] M. V. Patel, H. A. Scott, M. M. Marinak, in 52nd Annual Meeting of the American Physical Society Division of Plasma Physics, Vol. 55 (2010).

[46] H. A. Scott, S. B. Hansen, Advances in NLTE modeling for integrated simulations, High Energy Density Phys. 6 (1) (2010) $39-47$. doi:10.1016/j.hedp.2009.07.003. 
URL http://dx.doi.org/10.1016/j.hedp.2009.07.003

[47] D. A. Young, E. M. Corey, A new global equation of state model for hot, dense matter J. of Appl. Phys. 78 (6) (1995) 3748-3755. doi:10.1063/1.359955 URL http://dx.doi.org/10.1063/1.359955

[48] R. M. More, K. H. Warren, D. A. Young, G. B. Zimmerman, A new quotidian equation of state (QEOS) for hot dense matter Phys. of Fluids 31 (10) (1988) 3059-3078. doi:10.1063/1. 866963

URL http://dx.doi.org/10.1063/1.866963

[49] scikit-learn developers, Gaussian processes, http:// scikit-learn.org/stable/modules/gaussian_process.html 2016 (accessed 05.15.2017).

[50] Python Software Foundation, shelve - Python object persistence, https://docs.python.org/2/library/shelve.html 2017 (accessed 05.15.2017).

[51] The SciPy community, scipy.optimize.basinghopping, https://docs.scipy.org/doc/scipy-0.14.0/reference/ generated/scipy.optimize.basinhopping.html, 2009 (accessed 05.15.2017).

[52] The SciPy community, scipy.optimize.brute, https: //docs.scipy.org/doc/scipy-0.14.0/reference/generated/ scipy.optimize.brute.html, 2009 (accessed 05.15.2017).

[53] M. D. McKay, R. J. Beckman, W. J. Conover, A comparison of three methods for selecting values of input variables in the analysis of output from a computer code, Technometrics 21 (2) (1979) 239-245.

URL http://www. jstor.org/stable/1268522

[54] scikit-learn developers, $\mathrm{R}^{2}$ score, the coefficient of determination, http://scikit-learn. org/stable/modules/model_evaluation.html\# r2-score-the-coefficient-of-determination $2016 \quad$ (accessed 05.15.2017).

[55] K. Pearson, Note on regression and inheritance in the case of two parents Proc. of the R. Soc. of Lond. 58 (347-352) (1895) 240-242. URL http://dx.doi.org/10.1098/rspl.1895.0041

[56] E. W. Weisstein, $\mathrm{L}^{1}$-norm, from MathWorld-A Wolfram Web Resource.http://mathworld.wolfram.com/L1-Norm.html, 2017 (accessed 05.15.2017).

[57] T. Nagayama, J. E. Bailey, R. C. Mancini, C. A. Iglesias, S. B. Hansen, C. Blancard, H. K. Chung, J. Colgan, Ph. Cosse, G. Faussurier, R. Florido, C. J. Fontes, F. Gilleron, I. E. Golovkin, D. P. Kilcrease, G. Loisel, J. J. MacFarlane, J.-C. Pain, G. A. Rochau, M. E. Sherrill, R. W. Lee, Model uncertainties of local-thermodynamic-equilibrium K-shell spectroscopy, High Energy Density Phys. 20 (2016) $17-22$ URL https://doi.org/10.1016/j.hedp.2016.05.001

[58] H. Chen, R. Shepherd, H. K. Chung, A. Kemp, S. B. Hansen, S. C. Wilks, Y. Ping, K. Widmann, K. B. Fournier, G. Dyer, A. Faenov, T. Pikuz, P. Beiersdorfer, Fast-electron-relaxation measurement for laser-solid interaction at relativistic laser intensities Phys. Rev. E 76 (2007) 056402.

URL http://dx.doi.org/10.1103/PhysRevE.76.056402

[59] P. M. Nilson, A. A. Solodov, J. F. Myatt, W. Theobald, P. A. Jaanimagi, L. Gao, C. Stoeckl, R. S. Craxton, J. A. Delettrez, B. Yaakobi, J. D. Zuegel, B. E. Kruschwitz, C. Dorrer, J. H. Kelly, K. U. Akli, P. K. Patel, A. J. Mackinnon, R. Betti, T. C. Sangster, D. D. Meyerhofer, Scaling hot-electron generation to high-power, kilojoule-class laser-solid interactions Phys. Rev. Lett. 105 (2010) 235001 URL http://dx.doi.org/10.1103/PhysRevLett.105.235001

[60] P. M. Nilson, W. Theobald, C. Mileham, C. Stoeckl, J. F. Myatt, J. A. Delettrez, J. MacFarlane, I. A. Begishev, J. D. Zuegel, R. Betti, T. C. Sangster, D. D. Meyerhofer, Target-heating effects on the $\mathrm{K} \alpha_{1,2}$-emission spectrum from solid targets heated by laser-generated hot electrons, Phys. of Plasmas 18 (4) (2011) 042702. doi:10.1063/1.3574383

[61] P. M. Nilson, J. R. Davies, W. Theobald, P. A. Jaanimagi, C. Mileham, R. K. Jungquist, C. Stoeckl, I. A. Begishev, A. A. Solodov, J. F. Myatt, J. D. Zuegel, T. C. Sangster, R. Betti,
D. D. Meyerhofer, Time-resolved measurements of hot-electron equilibration dynamics in high-intensity laser interactions with thin-foil solid targets Phys. Rev. Lett. 108 (2012) 085002. doi:10.1103/PhysRevLett.108.085002

URL http://link.aps.org/doi/10.1103/PhysRevLett.108. 085002

[62] P. M. Nilson, A. A. Solodov, J. R. Davies, W. Theobald, C. Mileham, C. Stoeckl, I. A. Begishev, J. D. Zuegel, D. H. Froula, R. Betti, D. D. Meyerhofer, Time-resolved K $\alpha$ spectroscopy measurements of hot-electron equilibration dynamics in thin-foil solid targets: collisional and collective effects, J. of Phys. B: At., Mol. and Opt. Phys. 48 (22) (2015) 224001. doi: 10.1088/0953-4075/48/22/224001.

URL http://dx.doi .org/10.1088/0953-4075/48/22/224001

[63] C. R. Stillman, P. M. Nilson, S. T. Ivancic, C. Mileham, I. A. Begishev, R. K. Junquist, D. J. Nelson, D. H. Froula, A streaked x-ray spectroscopy platform for rapidly heated, near-solid density plasmas Rev. of Sci. Instrum. 87 (11) (2016) 11E312. URL http://dx.doi.org/10.1063/1.4960414 
Table 1: Design constraints at the time of peak temperature included in the general optimization problem

\begin{tabular}{|c|c|c|}
\hline Constraint no. & Design constraint description & Constraint form \\
\hline 1 & peak $T^{\text {peak }}$ is larger than $-5 \%$ of goal $T^{*}$ & $0.95-\frac{T^{\text {pak }}}{T^{*}} \leq 0$ \\
\hline 2 & peak $T^{\text {peak }}$ is less than $+5 \%$ of goal $T^{*}$ & $\frac{T^{\text {peak }}}{T^{*}}-1.05 \leq 0$ \\
\hline 3 & $\rho^{\text {peak }}$ is larger than minimum design $\rho^{*}$ & $1-\frac{\rho^{\text {peak }}}{\rho^{*}} \leq 0$ \\
\hline 4 & $\begin{array}{l}\text { period of constant } T \text { and } \rho\left(\Delta t^{\text {peak }}\right) \text { is larger than minimum } \\
\quad \text { design period }\left(\Delta t^{*}\right)\end{array}$ & $1-\frac{\Delta t^{\mathrm{peak}}}{\Delta t^{*}} \leq 0$ \\
\hline 5 & $\begin{array}{l}\text { variation in } T \text { across buried layer }\left(\Delta T^{\text {peak }}\right) \text { is less than max- } \\
\quad \text { imum design variation }\left(\Delta T^{*}\right)\end{array}$ & $\frac{\Delta T^{\text {peak }}}{\Delta T^{*}}-1 \leq 0$ \\
\hline 6 & $\begin{array}{l}\text { variation in } \rho \text { across buried layer }\left(\Delta \rho^{\text {peak }}\right) \text { is less than maxi- } \\
\text { mum design variation }\left(\Delta \rho^{*}\right)\end{array}$ & $\frac{\Delta \rho^{\text {peak }}}{\Delta \rho^{*}}-1 \leq 0$ \\
\hline 7 & optical depth $\left(\tau^{\text {peak }}\right)$ is larger than design minimum $\left(>\tau_{\min }^{*}\right)$ & $1-\frac{\tau^{\text {peak }}}{\tau_{\min }^{*}} \leq 0$ \\
\hline 8 & optical depth $\left(\tau^{\text {peak }}\right)$ is less than design maximum $\left(<\tau_{\max }^{*}\right)$ & $\frac{\tau^{\text {peak }}}{\tau_{\max }^{*}}-1 \leq 0$ \\
\hline 9 & $\begin{array}{l}\text { x-ray emission }\left(I_{\nu}^{\text {peak }}\right) \text { larger than minimum signal }\left(I_{\nu}^{*}\right) \text { re- } \\
\quad \text { quired for spectrometer }\end{array}$ & $1-\frac{I_{\nu}^{\text {peak }}}{I_{\nu}^{*}} \leq 0$ \\
\hline 10 & $\begin{array}{l}\text { dopant } \mathrm{H}-\alpha \text { to } \mathrm{He}-\alpha \text { line ratio }\left(R_{\alpha}^{\text {peak }}\right) \text { is larger than minimum } \\
\quad \text { design ratio }\left(>R_{\alpha}^{*, \text { min }}\right)\end{array}$ & $1-\frac{R_{\alpha}^{\text {peak }}}{R_{\alpha}^{*, \min }} \leq 0$ \\
\hline 11 & $\begin{array}{l}\text { dopant } \mathrm{H}-\alpha \text { to } \mathrm{He}-\alpha \text { line ratio }\left(R_{\alpha}^{\text {peak }}\right) \text { is less than maximum } \\
\quad \text { design ratio }\left(<R_{\alpha}^{*, \max }\right)\end{array}$ & $\frac{R_{\alpha}^{\text {peak }}}{R_{\alpha}^{*, \max }}-1 \leq 0$ \\
\hline 12 & $\begin{array}{l}\text { dopant } \mathrm{H}-\beta \text { to } \mathrm{He}-\beta \text { line ratio }\left(R_{\beta}^{\text {peak }}\right) \text { is larger than minimum } \\
\quad \text { design ratio }\left(>R_{\beta}^{*, \min }\right)\end{array}$ & $1-\frac{R_{\beta}^{\text {peak }}}{R_{\beta}^{* \text { min }}} \leq 0$ \\
\hline 13 & $\begin{array}{l}\text { dopant } \mathrm{H}-\beta \text { to } \mathrm{He}-\beta \text { line ratio }\left(R_{\beta}^{\text {peak }}\right) \text { is less than maximum } \\
\quad \text { design ratio }\left(<R_{\beta}^{*, \max }\right)\end{array}$ & $\frac{R_{\beta}^{\text {peak }}}{R_{\beta}^{*, \max }}-1 \leq 0$ \\
\hline 14 & $\begin{array}{l}\text { dopant } \mathrm{H}-\alpha \text { line emission signal }\left(S_{\mathrm{H}-\alpha}^{\mathrm{peak}}\right) \text { is larger than mini- } \\
\text { mum signal required for spectrometer }\left(>S_{\mathrm{H}-\alpha}^{*, \min }\right)\end{array}$ & $1-\frac{S_{\mathrm{H}-\alpha}^{\text {peak }}}{S_{\mathrm{H}-\alpha}^{*, \min }} \leq 0$ \\
\hline 15 & $\begin{array}{l}\text { dopant He- } \alpha \text { line emission signal }\left(S_{\mathrm{He}-\alpha}^{\text {peak }}\right) \text { is larger than min- } \\
\text { imum signal required for spectrometer }\left(>S_{\mathrm{He}-\alpha}^{*, \min }\right)\end{array}$ & $1-\frac{S_{\mathrm{He}-\alpha}^{\text {peak }}}{S_{\mathrm{He}-\alpha}^{*, \text { min }}} \leq 0$ \\
\hline 16 & $\begin{array}{l}\text { dopant } \mathrm{H}-\beta \text { line emission signal }\left(S_{\mathrm{H}-\beta}^{\mathrm{peak}}\right) \text { is larger than mini- } \\
\text { mum signal required for spectrometer }\left(>S_{\mathrm{H}-\beta}^{*, \min }\right)\end{array}$ & $1-\frac{S_{\mathrm{H}-\beta}^{\text {peak }}}{S_{\mathrm{H}-\beta}^{*, \min }} \leq 0$ \\
\hline 17 & $\begin{array}{l}\text { dopant } \mathrm{He}-\beta \text { line emission signal }\left(S_{\mathrm{He}-\beta}^{\mathrm{peak}}\right) \text { is larger than min- } \\
\text { imum signal required for spectrometer }\left(>S_{\mathrm{He}-\beta}^{*, \min }\right)\end{array}$ & $1-\frac{S_{\mathrm{He}-\beta}^{\mathrm{peak}}}{S_{\mathrm{He}-\beta}^{*, m i n}} \leq 0$ \\
\hline
\end{tabular}


Table 2: Design constraint values fixed for all design cases in this study

\begin{tabular}{|c|c|c|}
\hline Constraint no. & Design constraint value & Motivation for value \\
\hline 4 & $\Delta t^{*}=2.0 \mathrm{ps}$ & $\begin{array}{l}\text { consistent with temporal resolution of existing } \\
\text { x-ray streak spectrometers [4, 9, 63. }\end{array}$ \\
\hline 5 & $\Delta T^{*}=5 \%$ & $\begin{array}{l}\text { less than the uncertainty in inferred tempera- } \\
\text { ture in previous short pulse heated experi- } \\
\text { ments }[5,6,8,9,11\end{array}$ \\
\hline 6 & $\Delta \rho^{*}=10 \%$ & $\begin{array}{l}\text { less than the uncertainty in inferred density in } \\
\text { previous short pulse heated experiments }[5, \\
6,8,9,11\end{array}$ \\
\hline 7 & $\tau_{\min }^{*}=0.03$ & $\begin{array}{l}\text { selected to avoid regions of low opacity that } \\
\text { might cause emission to be too close to the } \\
\text { continuum emission }\end{array}$ \\
\hline 8 & $\tau_{\max }^{*}=0.64$ & $\begin{array}{l}\text { selected to limit the effect of optical depth on } \\
\text { the propagation of experimental uncertain- } \\
\text { ties }\end{array}$ \\
\hline 9 & $I_{\nu}^{*}=10^{13} \mathrm{~W} / \mathrm{cm}^{2} / \mathrm{str} / \mathrm{keV}$ & $\begin{array}{l}\text { selected to capture a minimum intensity that } \\
\text { could be measured on existing spectrometers } \\
{[8,9]}\end{array}$ \\
\hline 10 & $R_{\alpha}^{*, \min }=0.1$ & $\begin{array}{l}\text { selected to give a wide range of potential ratios } \\
\text { since parameter studies will explore a large } \\
\text { temperature regime }\end{array}$ \\
\hline 11 & $R_{\alpha}^{*, \max }=10.0$ & same as constraint 10 \\
\hline 12 & $R_{\beta}^{*, \min }=0.1$ & same as constraint 10 \\
\hline 13 & $R_{\beta}^{*, \max }=10.0$ & same as constraint 10 \\
\hline & $S_{\mathrm{H}-\alpha}^{*, \min }=10^{12} \mathrm{~W} / \mathrm{cm}^{2} / \mathrm{str}$ & $\begin{array}{l}\text { selected to match models for which the dopant } \\
\text { line begins to have significant signal }\end{array}$ \\
\hline 15 & $S_{\mathrm{He}-\alpha}^{*, \min }=7 \times 10^{11} \mathrm{~W} / \mathrm{cm}^{2} / \mathrm{str}$ & same as constraint 14 \\
\hline 16 & $S_{\mathrm{H}-\beta}^{*, \min }=6 \times 10^{11} \mathrm{~W} / \mathrm{cm}^{2} / \mathrm{str}$ & same as constraint 14 \\
\hline & $S_{\mathrm{He}-\beta}^{*, \min }=5 \times 10^{11} \mathrm{~W} / \mathrm{cm}^{2} / \mathrm{str}$ & same as constraint 14 \\
\hline
\end{tabular}

Table 3: Design constraint values for design cases considered

\begin{tabular}{|c|c|c|c|c|c|}
\hline Constraint no. & \multicolumn{2}{|c|}{$\begin{array}{l}\text { Design constraint values for the } \\
\text { upper radiative zone design set }\end{array}$} & \multicolumn{3}{|c|}{$\begin{array}{l}\text { Design constraint values for the } \\
\text { higher temperature design set }\end{array}$} \\
\hline $1 \& 2\left(T^{*}\right.$ in $\left.\mathrm{eV}\right)$ & 200 & 400 & 500 & 750 & 1000 \\
\hline $3\left(\rho^{*}\right.$ in $\left.\mathrm{g} / \mathrm{cm}^{3}\right)$ & 0.48 & 0.48 & 4.2 & 4.2 & 4.2 \\
\hline
\end{tabular}

Table 4: Optimized designs for the single parameter ensembles

\begin{tabular}{lrrrccc}
\hline Design case $(\mathrm{eV})$ & 200 & 300 & 400 & 500 & 750 & 1000 \\
\hline$E(\mathrm{~J})$ & 10.0 & 16.0 & 21.0 & 26.0 & 40.0 & 50.0 \\
$t_{\mathrm{FWHM}}(\mathrm{ps})$ & 1.2 & 0.7 & 0.7 & 0.01 & 0.01 & 0.01 \\
$\Delta l_{\mathrm{BL}}(\mu \mathrm{m})$ & 0.5 & 0.4 & 0.35 & 0.35 & 0.35 & 0.35 \\
$\Delta l_{\mathrm{CH}}(\mu \mathrm{m})$ & 3.8 & 2.6 & 1.8 & 1.0 & 0.5 & 0.5 \\
\hline
\end{tabular}

Table 5: Surrogate quality metrics for all design cases examined using the variable laser energy and buried layer thickness ensemble

\begin{tabular}{lrrrrrr}
\hline Design case (eV) & 200 & 300 & 400 & 500 & 750 & 1000 \\
\hline Number of training simulations & 691 & 691 & 691 & 636 & 636 & 622 \\
Number of testing simulations & 77 & 77 & 77 & 70 & 70 & 62 \\
$\mathrm{R}^{2}(\%)$ & 99.8 & 99.1 & 96.5 & 98.8 & 92.2 & 95.3 \\
Correlation score (\%) & 99.9 & 99.5 & 98.3 & 99.4 & 96.1 & 97.8 \\
L-1 relative error (\%) & 21.4 & 41.2 & 67.8 & 27.2 & 25.6 & 14.1 \\
\hline
\end{tabular}


Table 6: Optimized designs for the variable laser energy and buried layer thickness ensemble

\begin{tabular}{|c|c|c|c|c|c|c|}
\hline Design case $(\mathrm{eV})$ & 200 & 300 & 400 & 500 & 750 & 1000 \\
\hline$E(\mathrm{~J})$ & 12.8 & 17.5 & 20.6 & 27.5 & 38.6 & 55.8 \\
\hline$\Delta l_{\mathrm{BL}}(\mu \mathrm{m})$ & 0.17 & 0.22 & 0.26 & 0.26 & 0.28 & 0.41 \\
\hline$\sigma_{\kappa_{\nu}}^{\mathrm{rel}}(\%)$ & 39 & 32.1 & 13 & 20 & 20 & 15 \\
\hline Met $T^{*} ?$ & $37 \%$ larger & $23 \%$ larger & Yes & Yes & Yes & Yes \\
\hline Met $\rho^{*} ?$ & Yes & Yes & Yes & Yes & Yes & Yes \\
\hline Met $\Delta t^{*} ?$ & No & No & No & No & No & No \\
\hline Met $\Delta T^{*}$ and $\Delta \rho^{*}$ ? & $\Delta \rho=15 \%$ & $\Delta \rho=15 \%$ & $\Delta \rho=14 \%$ & $\Delta \rho=16 \%$ & $\Delta \rho=17 \%$ & $\Delta \rho=18 \%$ \\
\hline Within $\tau_{\min }^{*}$ and $\tau_{\max }^{*} ?$ & $\max =0.85$ & Yes & Yes & $\min =0.02$ & $\begin{array}{r}\min =0.01 \\
\max =1\end{array}$ & $\min =0.01$ \\
\hline Met $I_{\nu}^{*} ?$ & Yes & Yes & Yes & Yes & Yes & Yes \\
\hline Within $R^{*, \min }$ and $R^{*, \max } ?$ & No & No & No & $\begin{array}{l}\text { Only } \\
\quad \alpha \text { ratio }\end{array}$ & Yes & Yes \\
\hline Met $S^{*, \min }$ ? & No & $\begin{array}{l}\text { Only } \\
\text { He- } \alpha\end{array}$ & $\begin{array}{l}\text { Only } \\
\text { He- } \alpha\end{array}$ & $\begin{array}{l}\text { Only } \\
\text { He lines }\end{array}$ & Yes & Yes \\
\hline Cost function value & 32 & 20 & 9 & 7.5 & 9.2 & 7.5 \\
\hline
\end{tabular}

Table 7: Optimized designs for the variable laser energy and buried layer thickness ensemble with revised dopant materials

\begin{tabular}{llll}
\hline Design case $(\mathrm{eV})$ & 200 & 300 & $400 \mathrm{eV}$ \\
\hline$E(\mathrm{~J})$ & 12.8 & 17.5 & 20.6 \\
Dopant & $\mathrm{Mg}$ & $\mathrm{Al}$ & $\mathrm{Al}$ \\
$\Delta l_{\mathrm{BL}}(\mu \mathrm{m})$ & 0.17 & 0.22 & 0.26 \\
$\left.\sigma_{\kappa_{\nu}}^{\text {rel }} \%\right)$ & 22 & 26 & 12 \\
Met $T^{*} ?$ & $43 \%$ larger & $18 \%$ larger & Yes \\
Met $\rho^{*}$ Met $\Delta t^{*}$ Met $\Delta T^{*}$ and $\Delta \rho^{*} ?$ & Yes & Yes & Yes \\
Within $\tau_{\text {min }}^{*}$ and $\tau_{\max }^{*} ?$ & No & No & $\Delta \rho=15 \%$ \\
Met $I_{\nu}^{*}$ Within $R^{*, \text { min }}$ and $R^{*, \max ?}$ & $\Delta \rho=11 \%$ & $\Delta \rho=17 \%$ & $\max =0.95$ \\
Met $S^{*, \text { min? }}$ & $\max =0.7$ & $\max =0.96$ & Yes \\
Cost function value & Yes & Yes & Yes \\
\hline
\end{tabular}

Table 8: Surrogate quality metrics for all design cases examined using the variable laser energy, buried layer thickness, and tamper thickness ensemble

\begin{tabular}{lrrrrrr}
\hline Design case (eV) & 200 & 300 & 400 & 500 & 750 & 1000 \\
\hline Number of training simulations & 2139 & 2390 & 2505 & 2553 & 5288 & 5676 \\
Number of testing simulations & 238 & 265 & 278 & 284 & 661 & 631 \\
$\mathrm{R}^{2}(\%)$ & 98.8 & 98.4 & 94.8 & 95.4 & 95 & 93.9 \\
Correlation score (\%) & 99.4 & 99.2 & 97.4 & 97.7 & 97.5 & 96.9 \\
L-1 relative error (\%) & 22.3 & 46.4 & 63.6 & 65.3 & 64 & 59.3 \\
\hline
\end{tabular}

Table 9: Local minima identified by optimization methods for the $300 \mathrm{eV}$ design case.

\begin{tabular}{lcccc}
\hline & minimum 1 & minimum 2 & minimum 3 & minimum 4 \\
\hline Optimization method & brute force & basin hopping & basin hopping & basin hopping \\
$E(\mathrm{~J})$ & 0.11 & 12.6 & 54.8 & 41.5 \\
$\Delta l_{\mathrm{BL}}(\mu \mathrm{m})$ & 0.99 & 0.18 & 0.76 & 0.25 \\
$\Delta l_{\mathrm{CH}}(\mu \mathrm{m})$ & 5.8 & 2.5 & 8.8 & 8.9 \\
Cost function value & 368 & 18.7 & 24.4 & 27.7 \\
Cost function surrogate value & -118 & -90.6 & -8.45 & -40.1 \\
\hline
\end{tabular}


Table 10: Optimized designs for the variable laser energy, buried layer thickness, and tamper thickness ensemble with revised dopant materials

\begin{tabular}{|c|c|c|c|c|c|c|}
\hline Design case $(\mathrm{eV})$ & 200 & 300 & 400 & 500 & 750 & 1000 \\
\hline$E(\mathrm{~J})$ & 30.0 & 12.6 & 20.2 & 25.9 & 30.9 & 60.3 \\
\hline Dopant & $\mathrm{Mg}$ & $\mathrm{Al}$ & $\mathrm{Al}$ & $\mathrm{S}$ & $\mathrm{S}$ & $\mathrm{S}$ \\
\hline$\Delta l_{\mathrm{BL}}(\mu \mathrm{m})$ & 0.21 & 0.18 & 0.19 & 0.21 & 0.22 & 0.59 \\
\hline$\Delta l_{\mathrm{CH}}(\mu \mathrm{m})$ & 9.2 & 2.5 & 2.7 & 2.8 & 2.4 & 3 \\
\hline$\sigma_{\kappa}^{\mathrm{rel}}(\%)$ & 31 & 11 & 13 & 22 & 21 & 15 \\
\hline Met $T^{*}$ ? & $31 \%$ larger & Yes & $6 \%$ larger & $8 \%$ larger & Yes & Yes \\
\hline Met $\rho^{*} ?$ & Yes & Yes & Yes & $11 \%$ lower & Yes & Yes \\
\hline Met $\Delta t^{*} ?$ & No & No & No & No & No & No \\
\hline Met $\Delta T^{*}$ and $\Delta \rho^{*}$ ? & Yes & $\Delta \rho=16 \%$ & $\Delta \rho=17 \%$ & $\Delta \rho=17 \%$ & $\Delta \rho=17 \%$ & $\Delta \rho=13 \%$ \\
\hline Within $\tau_{\min }^{*}$ and $\tau_{\max }^{*} ?$ & $\max =1.9$ & $\max =0.94$ & Yes & $\min =0.02$ & $\min =0.01$ & $\begin{array}{l}\min =0.01 \\
\max =0.77\end{array}$ \\
\hline Met $I_{\nu}^{*}$ ? & Yes & Yes & Yes & Yes & Yes & Yes \\
\hline Within $R^{*, \min }$ and $R^{*, \max }$ ? & Not $\alpha$ ratio & Not $\alpha$ ratio & Yes & Yes & Yes & Yes \\
\hline Met $S^{*, \min }$ ? & Yes & Not $\mathrm{H}-\alpha$ & Yes & Yes & Yes & Yes \\
\hline Cost function value & 24 & 5.8 & 5.6 & 8.6 & 9 & 6.6 \\
\hline
\end{tabular}

